\title{
Essential Oils as an Alternative to Pyrethroids' Resistance against Anopheles Species Complex Giles (Diptera: Culicidae)
}

\author{
Olivier Gnankiné ${ }^{1}$ and Imaël Henri Nestor Bassolé ${ }^{2, *}$ \\ 1 Laboratoire d'entomologie fondamentale et appliquée (Lefa), Université Ouaga I Pr Joseph KI-ZERBO, \\ 03 P.O. 7021 Ouagadougou, Burkina Faso; olignankine@gmail.com or olivier.gnankine@univ-ouaga.bf \\ 2 Laboratoire de biologie moléculaire, d'épidémiologie et de surveillance des bactéries et virus transmis par \\ les aliments (Labesta), Université Ouaga I Pr Joseph KI-ZERBO, 03 P.O. 7021 Ouagadougou, Burkina Faso \\ * Correspondence: ismael.bassole@gmail.com; Tel.: +226-7812-5004
}

Received: 17 July 2017; Accepted: 1 August 2017; Published: 22 September 2017

\begin{abstract}
Widespread resistance of Anopheles sp. populations to pyrethroid insecticides has led to the search for sustainable alternatives in the plant kingdom. Among many botanicals, there is great interest in essential oils and their constituents. Many researchers have explored essential oils (EOs) to determine their toxicity and identify repellent molecules that are effective against Anopheles populations. Essential oils are volatile and fragrant substances with an oily consistency typically produced by plants. They contain a variety of volatile molecules such as terpenes and terpenoids, phenol-derived aromatic components and aliphatic components at quite different concentrations with a significant insecticide potential, essentially as ovicidal, larvicidal, adulticidal, repellency, antifeedant, growth and reproduction inhibitors. The current review provides a summary of chemical composition of EOs, their toxicity at different developmental stages (eggs, larvae and adults), their repellent effects against Anopheles populations, for which there is little information available until now. An overview of antagonist and synergistic phenomena between secondary metabolites, the mode of action as well as microencapsulation technologies are also given in this review. Finally, the potential use of EOs as an alternative to current insecticides has been discussed.
\end{abstract}

Keywords: essential oils; Anopheles sp.; insecticides; repellent; pyrethroid resistance

\section{Introduction}

Malaria, caused mainly by Anopheles mosquitoes, remains a major concern despite many efforts undertaken in vector control strategies. About $90 \%$ of malaria deaths worldwide in 2015 were registered in African countries, including the death of a child under five years old every $2 \mathrm{~min}$ [1].

Vector control relies primarily on two interventions in the form of long-lasting insecticidal nets (LLINs) and indoor residual spraying (IRS) [2,3]. This combination has saved thousands of lives during the past 10 years [2]. Pyrethroids, organochlorines (dichlorodiphenyltrichloroethane, DDT), organophosphates and carbamates are the active insecticidal ingredients recommended by World Health Organization (WHO) for IRS, while pyrethroids were the only products used for LLINs. These insecticides are known to be neurotoxic. Unfortunately, resistance to most pyrethroids used against adult mosquito populations in public health treatments has been detected in many countries and is now widespread and reported in two thirds of the countries with ongoing malaria transmission problems [4-7].

According to Silva et al. [8], pyrethroid resistance genes, termed as "knock down resistance" $(k d r)$ have already been found in at least 13 species (Anopheles gambiae, An. arabiensis, An. sinensis, An. stephensi, An. subpictus, An. sacharovi, An. culicifacies, An. sundaicus, An. aconitus, An. vagus, An. paraliae, An. peditaeniatus and An. albimanus) from mosquito populations on the African, Asian 
and, more recently, American continents. Seven mutational variants (L1014F, L1014S, L1014C, L1014W, N1013S, N1575Y and V1010L) have been described, with the highest prevalence of L1014F, which occurs at the 1014 site in channel sodium IIS6 domain [8]. In West Africa, pyrethroid resistance in Anopheles mosquitoes is mainly attributed to mutations of the sodium channel target site, the L1014F kdr [8-16]. To date, three mutations L1014F known as $k d r$-West, L1014S as $k d r$-East and N1575Y coexist in some countries and are widely distributed throughout Benin, Cameroon and Burkina Faso [7,8,17-21]. Up to now, in most countries, the $k d r$ genes are almost fixed in mosquito populations due mainly to high selection pressure exerted on adults directly but also indirectly in case of pest control in agriculture. It is important to know that chemicals currently used in control of agricultural pests are also the same ones used for vector control, therefore they are source of increasing the potential for resistance selection in mosquitoes as in An. gambiae [6].

Human health risks associated with the use of chemicals have led to the growth of an environmental movement seeking sustainable alternatives in pest control. Therefore, in recent years, various workers have been concentrating their efforts on the search for natural products derived from plants as an alternative to conventional insecticides used in controlling vectors for which resistance was detected [22]. Among many natural products, essential oils (EOs) and their constituents have received considerable attention in the search for new pesticides, and have been found to possess an insecticidal potential [23]. These natural compounds are generally recognized as safe (GRAS) for the environment and human health, which explains our interest in their use for a sustainable agriculture and human health.

According to Isman [24], most essential oils (EOs) and their major constituents are relatively non-toxic to mammals, with acute oral Lethal Dose (LD) values in rodents ranging from 800 to $3000 \mathrm{mg} \cdot \mathrm{kg}^{-1}$ for pure compounds and $>>5000 \mathrm{mg} \cdot \mathrm{kg}^{-1}$ for formulated products. Many EOs show toxic effects against several insect species due to their multiple modes and sites of action in the insects' nervous system [25,26]. Most widely known plants used for protection against mosquitoes belong to the families Myrtaceae, Lauraceae, Rutaceae, Lamiaceae, Asteraceae, Apiaceae, Cupressaceae, Poaceae, Zingeberaceae and Piperaceae [25]. According to Nerio [27], it is important to test some parameters of EOs such as their human toxicity, before promoting their use. Although some of them such as citronella, lemon and eucalyptus oils are recommended by the U.S. Environmental Protection Agency (US EPA) as repellent ingredients for application on the skin due to their relative low toxicity, comparable efficiency and customer approval, others might possess a higher toxicity than chemicals and thus cause skin irritation [28].

Essential oils are oily aromatic liquids extracted from plants [29]. Techniques commonly employed for their extraction include hydrodistillation, steam distillation, solvent extraction, head space analysis and liquid $\mathrm{CO}_{2}$ extraction [30-33].

The review focuses on the examination of the recent knowledge concerning the use of essential oils and their constituents against Anopheles mosquitoes, the main vector of malaria diseases, for which little information is available. Bioactive components' interactions, their mode of action and microencapsulation technologies are also addressed in this survey.

\section{Chemical Composition}

Essential oils are natural, complex, multi-component mixture including hydrocarbons (terpenes), oxygenated hydrocarbons (terpenoids), and phenylpropenes [34]. Both terpenes and terpenoids are based on the 2-methylbuta-1,3-diene $\left(\mathrm{C}_{5} \mathrm{H}_{8}\right)$ unit called isoprene.

The isoprene unit builds up in repeating units to form monoterpenes, sesquiterpenes, diterpenes, triterpenes and tetraterpenes containing 2, 3, 4, 6 and 8 isoprene units, respectively. Only monoterpenes, sesquiterpenes and diterpenes are found in essential oils. Monoterpenes together with sesquiterpenes are the most abundant constituents of essential oils [35]. They are either acyclic or cyclic. Terpenoids are terpenes with alcohol, phenol, aldehyde, ketone or ester functional groups (Figure 1). Phenylpropenes are compounds based on a phenylpropane skeleton and many are derived from a biochemical pathway called the shikimic acid pathway. 


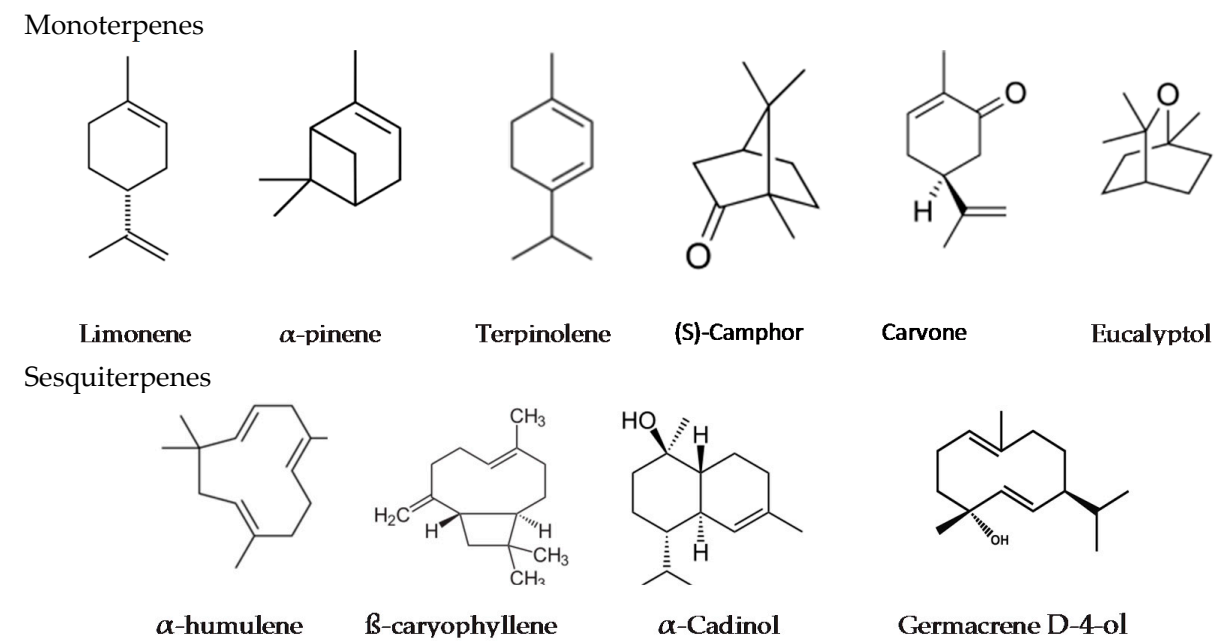

Phenylpropenes<smiles>O=C/C=C/c1ccccc1</smiles><smiles>C=CCc1ccc(O)c(OC)c1</smiles><smiles>C=CCc1ccc2c(c1)OCO2</smiles>

Cinnamaldehyde

Eugenol

Safrole

Figure 1. Structural formulae of selected essential oil components.

\section{Toxicity to Eggs and Immature Stages in Anopheles sp.}

Many plant essential oils are reported to possess ovicidal, larvicidal and pupacidal activities against malaria vectors according to the WHO test procedures [36-39]. Research reports show that essential oils have ovicidal activities against Anopheles gambiae s.l., An. gambiae s.s. [37], and An. stephensi (Table 1) $[36,37]$.

Table 1. Toxicity of essential oils against eggs of Anopheles sp.

\begin{tabular}{|c|c|c|c|c|c|c|c|}
\hline Plant Species & $\begin{array}{l}\text { Plant } \\
\text { Organ }\end{array}$ & $\begin{array}{c}\text { Extraction } \\
\text { Method }\end{array}$ & Species & $\begin{array}{l}\mathrm{LC}_{50} \\
(\mathrm{mg} / \mathrm{L})\end{array}$ & $\begin{array}{c}\mathrm{LC}_{90} \\
(\mathrm{mg} / \mathrm{L})\end{array}$ & $\begin{array}{c}\mathrm{LC}_{95} \\
(\mathrm{mg} / \mathrm{L})\end{array}$ & Reference \\
\hline Cinnamomum zeylanicum & Bark & Hydrod. & An. stephensi & NI & NI & 130.0 & [36] \\
\hline Сuminum сутіпит & Seed & Hydrod. & An. stephensi & NI & NI & 150.7 & [36] \\
\hline Curcuma longa & Rhizome & Hydrod. & An. stephensi & NI & NI & 89.9 & [36] \\
\hline Juniperus macropoda & Fruit & Hydrod. & An. stephensi & NI & NI & 59.3 & [36] \\
\hline Ocimum basilicum & Leaf & Hydrod. & An. stephensi & NI & NI & 172.8 & [36] \\
\hline Pimpinella anisum & Seed & Hydrod. & An. stephensi & NI & NI & 33.3 & [36] \\
\hline Zingiber officinalis & Rhizome & Hydrod. & An. stephensi & NI & NI & 32.2 & [36] \\
\hline Lippia multiflora & Leaf & Hydrod. & An. gambiae s.s & 17.1 & 33.5 & NI & [37] \\
\hline Ocimum canum & Leaf & Hydrod. & An. gambiae s.s & 188.7 & 488.0 & NI & [37] \\
\hline Cymbopogon proximus & Leaf & Hydrod. & An. gambiae s.s & 52.8 & 91.1 & NI & [37] \\
\hline
\end{tabular}

Hydrod. = Hydrodistillation; $\mathrm{LC}_{50}, \mathrm{LC}_{90}$ and $\mathrm{LC}_{95}$ are the lethal concentrations that kill $50 \%, 90 \%$ and $95 \%$ of eggs, respectively; NI: No information is available.

Bassolé et al. [37] tested the efficiency of Cymbopogon proximus, Lippia multiflora and Ocimum canum essential oils against $A n$. gambiae eggs and found that the $\mathrm{LC}_{50}$ values ranged from 61.9 to $188.8 \mathrm{mg} \cdot \mathrm{L}^{-1}$. Eggs of An. stephensi were susceptible to the essential oils of Cinnamomum zeylanicum, Cuminum cyminum, Curcuma longa, Juniperus macropoda, Ocimum basilicum, Pimpinella anisum, Zingiber officinalis and Cinnamomum zeylanicum. $\mathrm{LC}_{95}$ varied from 32.2 to $172.8 \mathrm{mg} \cdot \mathrm{L}^{-1}$ [36]. 
Table 2. Toxicity of essential oils against larvae of Anopheles sp.

\begin{tabular}{|c|c|c|c|c|c|c|c|c|}
\hline Plant Species & Plant Organ & $\begin{array}{l}\text { Extraction } \\
\text { Method }\end{array}$ & Mosquito & $\begin{array}{c}\mathrm{LC}_{50} \\
(\mathrm{mg} / \mathrm{L})\end{array}$ & $\begin{array}{c}\mathrm{LC}_{90} \\
(\mathrm{mg} / \mathrm{L})\end{array}$ & $\begin{array}{c}\mathrm{LC}_{95} \\
(\mathrm{mg} / \mathrm{L})\end{array}$ & $\begin{array}{c}\mathrm{LC}_{99} \\
(\mathrm{mg} / \mathrm{L})\end{array}$ & Ref. \\
\hline Amomum subulatum & Leaf & Hydrod. & An. subpictus & 41.2 & 80.3 & NI & NI & [40] \\
\hline Apium graveolens & Whole plant & Steam-d. & An. dirus & 59.4 & & 111.7 & 159.1 & [41] \\
\hline Artemisia gilvescens & Plant & Hydrod. & An. anthropophagus & 49.9 & 97.4 & NI & NI & [42] \\
\hline Artemisia gilvescens & Whole & Hydrod. & An. anthropophagus & 49.0 & 97.0 & NI & NI & [42] \\
\hline Blumea densiflora & Aerial parts & Hydrod. & An. anthropophagus & 10.0 & 33.0 & NI & NI & [42] \\
\hline Bunium persicum & Seed & Hydrod. & An. stephensi & 27.7 & 91.3 & NI & NI & [43] \\
\hline Carum carvi & Whole plant & Steam-d. & An. dirus & 72.2 & NI & 104.7 & 128.7 & [41] \\
\hline Chenopodium ambrosioides & Aerial parts & Hydrod. & An. arabiensis & 17.5 & NI & NI & NI & [44] \\
\hline Chloroxylon swietenia & Leaf and stem & Hydrod. & An. stephensi & 14.0 & 22.0 & NI & NI & [45] \\
\hline Cionura erecta & Root & Hydrod. & An. stephensi & 77.0 & 199.0 & NI & NI & [46] \\
\hline Citrus aurantium & Fruit & Hydrod. & A. stephensi & 31.2 & 73.8 & NI & NI & [47] \\
\hline Citrus paradisi & Fruit & Hydrod. & An. stephensi & 35.7 & 70.2 & NI & NI & [47] \\
\hline Coleus aromaticus & Leaf & Hydrod. & An. subpictus & 60.3 & 118.7 & NI & NI & [48] \\
\hline Cryptomeria japonica & Leaf & Hydrod. & An. gambiae & 67.1 & 193.7 & NI & NI & [49] \\
\hline Curcuma longa & Leaf & Hydrod. & An. quadrimaculatus & 1.8 & 4.1 & NI & NI & [50] \\
\hline Curcuma longa & Rhizome & Hydrod. & An. quadrimaculatus & 3.7 & 9.4 & NI & NI & [50] \\
\hline Curcuma zedoaria & Whole plant & Steam-d. & An. dirus & 29.7 & NI & 40.2 & 47.7 & [41] \\
\hline Cymbopogon citratus & Leaf & Hydrod. & An. funestus & 34.6 & NI & NI & NI & [51] \\
\hline Cymbopogon citratus & Leaf & Hydrod. & An. gambiae & 18.0 & NI & NI & NI & [38] \\
\hline Cymbopogon proximus & Leaf & Hydrod. & An. gambiae & 69.7 & NI & NI & NI & [37] \\
\hline Feronia limonia & Leaf & Hydrod. & An. stephensi & 15.0 & 36.7 & NI & NI & [52] \\
\hline Ferulago carduchorum & Aerial parts & Hydrod. & An. stephensi & 12.0 & 47.0 & NI & NI & [53] \\
\hline Ferulago carduchorum & Aerial part & Hydrod. & An. stephensi & 12.0 & 47.0 & NI & NI & [53] \\
\hline Foeniculum vulgare & Whole plant & Steam-d. & An. dirus & 35.3 & NI & 38.8 & 40.9 & [41] \\
\hline Juniperus procera & Leaf & Hydrod. & An. arabiensis & 24.5 & 34.2 & $\mathrm{NI}$ & $\mathrm{NI}$ & [54] \\
\hline Lavandula gibsoni & Plant & Hydrod. & An. stephensi & 62.8 & 129.0 & NI & NI & [55] \\
\hline Lippia multiflora & Leaf & Hydrod. & An. gambiae & 61.9 & NI & NI & NI & [37] \\
\hline Mentha spicata & Aerial part & Hydrod. & An. stephensi & 49.7 & 101.0 & NI & NI & [56] \\
\hline Mentha spicata & Leaf & Hydrod. & An. stephensi & 82.9 & NI & NI & NI & [57] \\
\hline Murraya exotica & Leaf & Hydrod. & An. stephensi & 56.3 & 107.8 & NI & NI & [58] \\
\hline Nigella sativa & Leaf & Hydrod. & An. arabiensis & 23.4 & NI & NI & NI & [44] \\
\hline Ocimum basilicum & Leaf & Hydrod. & An. subpictus & 9.75 & 18.6 & NI & NI & [56] \\
\hline Ocimum canum & Leaf & Hydrod. & An. funestus & 91.2 & NI & NI & NI & [51] \\
\hline Ocimum lamiifolium & Leaf & Hydrod. & An. arabiensis & 20.9 & NI & NI & NI & [44] \\
\hline Origanum vulgare & Leaf & Hydrod. & An. subpictus & 67.0 & 128.6 & NI & NI & [59] \\
\hline Piper capense & Plant & Hydrod. & An. gambiae & 34.9 & 85.0 & NI & NI & [60] \\
\hline Plectranthus amboinicus & Leaf & Hydrod. & An. gambiae & 55.2 & 99.1 & NI & NI & [61] \\
\hline Plectranthus amboinicus & Leaf & Hydrod. & An. stephensi & 28.37 & 59.4 & NI & NI & [62] \\
\hline Plectranthus barbatus & Leaf & Hydrod. & An. subpictus & 84.2 & 165.2 & NI & NI & [63] \\
\hline Plectranthus mollis & Whole plant & Hydrod. & An. stephensi & 33.5 & NI & NI & NI & [55] \\
\hline Ruta chalepensis & Aerial parts & & An. quadrimaculatus & 15.0 & 42.0 & NI & NI & [64] \\
\hline Salvia elegan & Aerial parts & Hydrod. & An. quadrimaculatus & 6.2 & 15.8 & NI & NI & [50] \\
\hline Salvia leucantha & Aerial parts & Hydrod. & An. quadrimaculatus & 10.9 & 29.1 & NI & NI & [50] \\
\hline Salvia officinalis & Aerial parts & Hydrod. & An. quadrimaculatus & 14.1 & 35.8 & NI & NI & [50] \\
\hline Schinus molle & Leaf and seed & Hydrod. & An. arabiensis & 21 & NI & NI & NI & [44] \\
\hline Syzygium zeylanicum & Leaf & Hydrod. & An. subpictus & 83.1 & 164.2 & NI & NI & [65] \\
\hline Tagetes patula & Leaf & Steam-d. & An. stephensi & 12.0 & 57.0 & NI & NI & [66] \\
\hline Toddalia asiatica & Root & Hydrod. & An. stephensi & 69.0 & 110.0 & NI & NI & [67] \\
\hline Trachyspermum ammi & Seed & Steam-d. & An. stephensi & 80.8 & NI & NI & 172.1 & [68] \\
\hline Zanthoxylum armatum & Seed & Hydrod. & An. stephensi & 58.0 & 183.0 & NI & NI & [69] \\
\hline Zanthoxylum limonella & Whole plant & Steam-d. & Anopheles dirus & 57.2 & NI & 76.2 & 89.5 & [41] \\
\hline Zhumeria majdae & Leaf & Hydrod. & An. stephensi & 61.3 & 135.8 & NI & NI & [70] \\
\hline Zingiber nimmoni & Rhizome & Hydrod. & An. stephensi & 41.2 & 80.3 & NI & NI & [59] \\
\hline
\end{tabular}

Hydrod. = Hydrodistillation; Steam-d. = Steam distillation; $\mathrm{LC}_{50}, \mathrm{LC}_{90}$ and $\mathrm{LC}_{95}$ are the lethal concentrations that kill $50 \%, 90 \%$ and $95 \%$ of eggs, respectively; NI: No information is available. 
Table 3. Toxicity of essential oils components against Anopheles sp. larvae.

\begin{tabular}{|c|c|c|c|c|}
\hline Components of EO & Species & $\mathrm{LC}_{50}(\mathrm{mg} / \mathrm{L})$ & $\mathrm{LC}_{90}(\mathrm{mg} / \mathrm{L})$ & Reference \\
\hline \multicolumn{5}{|c|}{ Monoterpene hydrocarbons } \\
\hline (+)-Limonene & An. gambiae s.s. & 270.3 & NI & [71] \\
\hline Limonene & An. stephensi & 8.8 & 17.6 & [72] \\
\hline$\alpha$-Pinene & An. subpictus & 32.1 & 62.8 & [73] \\
\hline Terpinolene & An. gambiae s.s. & 404.71 & NI & [71] \\
\hline Camphor & An. anthropophagus & 129.7 & 192.4 & [42] \\
\hline Carvacrol & An. subpictus & 21.1 & 41.9 & [65] \\
\hline Carvone & An. stephensi & 19.3 & 37.1 & [72] \\
\hline (-)-Carvone epoxide & Anopheles gambiae s.s. & 124.7 & NI & [71] \\
\hline cis-Carveol & An. stephensi & 28.5 & 59.2 & [72] \\
\hline Eucalyptol & An. anthropophagus & $>200$ & NI & [42] \\
\hline (-)-Hydroxycarvone & An. gambiae s.s. & 1172.2 & NI & [71] \\
\hline (-)-Isopulegol (2) & An. gambiae s.s. & 49.4 & NI & [71] \\
\hline (+)-Limonene epoxide & An. gambiae s.s. & 200.8 & NI & [71] \\
\hline (-)-Perillyl alcohol & An. gambiae s.s. & 18.4 & NI & [71] \\
\hline Piperitenone oxide & An. stephensi & 25.8 & NI & [57] \\
\hline Terpine-4-ol & An. anthropophagus & 76.7 & 139.4 & [42] \\
\hline Terpinen-4-ol & An. gambiae s.s. & 337.7 & NI & [71] \\
\hline Terpinen-4-ol & An. subpictus & 43.3 & 84.1 & [65] \\
\hline Thymol & An. stephensi & 48.88 & NI & [68] \\
\hline Thymol & An. subpictus & 22.06 & 40.0 & [48] \\
\hline \multicolumn{5}{|c|}{ Sesquiterpenes } \\
\hline$\alpha$-Humulene & An. subpictus & 6.19 & 12.0 & [65] \\
\hline$\beta$-Caryophyllene & An. subpictus & 41.66 & 84.9 & {$[65]$} \\
\hline$\beta$-Elemene & An. subpictus & 10.26 & 20.0 & [65] \\
\hline Caryophyllene & An. anthropophagus & $>200$ & & [42] \\
\hline Germacrene D & An. anthropophagus & 49.81 & 106.2 & [42] \\
\hline$\alpha$-Cadinol & An. subpictus & 10.27 & 20.4 & [74] \\
\hline Ar-turmerone & An. quadrimaculatus & 2.8 & 7.0 & [50] \\
\hline Caryophyllene oxide & An. anthropophagus & 49.46 & 115.4 & [42] \\
\hline Germacrene D-4-ol & An. subpictus & 6.12 & 12.1 & [74] \\
\hline \multicolumn{5}{|c|}{ Phenylpropenes } \\
\hline Eugenol & An. subpictus & 25.45 & 50.6 & [65] \\
\hline \multicolumn{5}{|c|}{ Others } \\
\hline Curcumin & An. quadrimaculatus & 32.5 & 74.6 & {$[50]$} \\
\hline Desmethoxycurcumin & An. quadrimaculatus & 29.7 & 66.7 & [50] \\
\hline
\end{tabular}

$\mathrm{LC}_{50}$ and $\mathrm{LC}_{90}$ are the lethal concentrations that kill $50 \%$ and $90 \%, 95 \%$ and $99 \%$ of larvae, respectively.

According to Dias and Moraes [75], plant essential oils were considered active when their LC $_{50}$ values were below $100 \mathrm{mg} \cdot \mathrm{L}^{-1}$ against larvae vector mosquitoes such as Aedes sp. Thus, essential oils from 63 plant species demonstrated larvicidal activity against Anopheles anthropophagus, An. atroparvus, An. arabiensis, An. dirus, An. funestus, An. gambiae s.l., An. gambiae s.s., An. quadrimaculatus, An. stephensis and An. subpictus (Table 2). Table 2 shows that $\mathrm{LC}_{50}$ values were between 1.8 and $91.2 \mathrm{mg} \cdot \mathrm{L}^{-1}$ whereas $\mathrm{LC}_{90}$ were from 4.1 to $199 \mathrm{mg} \cdot \mathrm{L}^{-1}$. Many researchers have shown the efficiency of major compounds from EOs on Anopheles sp. larvae (Table 3). Essential oil from Curcuma longa was the most toxic against An. quadrimaculatus larva. All values are above the World Health Organization (WHO) guideline of $1 \mathrm{mg} \cdot \mathrm{L}^{-1}$ for larvicides. The use of EOs extracted from plants seems to be problematic with regards to their $\mathrm{LC}_{50}$ values. However, these studies should be interesting, if the tests carried out take into account the susceptibility of reducing the use of the chemicals commonly used in human protection. 


\section{Essential Oils Toxicity in Anopheles sp. Adults}

In addition to their effects on eggs and larvae, EOs exhibit toxic effects on adults of malaria vectors following WHO test kits and topical applications recommended by WHO $[36,39,76,77]$ (Table 4). Recently, Deletre et al. [39] tested the toxic effects of 20 plant extracts on the adults of the malaria vector An. gambiae. Amongst them, the most promising plant extracts are those from Cymbopogon winterianus, Cinnamomum zeylanicum and Thymus vulgaris. The mortality due to these EOs was higher than that observed with permethrin. In Benin, Bossou et al. [77] investigated on the effect of nine EOs extracted from plants on susceptible strain "Kisumu". The plants tested were Chenopodium ambrosioides L., Securidaca longepedunculata Fresen., Cochlospermum planchonii Hook. f. Ex Planch., Cochlospermum tinctorium A. Rich., Eucalyptus tereticornis Sm., Eucalyptus citriodora Hook. Cymbopogon citratus (DC.) Stapf, Cymbopogon schoenanthus (L.) Spreng. and Cymbopogon giganteus Chiov. They showed that mortality rate of An. gambiae "Kisumu" varies depending on the concentration. The most efficient essential oil was C. citratus, followed by E. tereticornis, E. citriodora and C. ambrosioides, C. schoenanthus, C. giganteus, C. planchonii and S. longepedunculata (Table 4). These oils showed 100\% mortality rates. For instance, the threshold of susceptibility was fixed at $98 \%$ for the active molecules according to the WHO protocol [78]. The resistance/susceptibility status was evaluated according to WHO [78] criteria, considering mortality above $97 \%$ and below $90 \%$ representative of susceptibility and resistance, respectively, but between the two values, resistance should be suspected.

An. gambiae resistant strains were susceptible to all essential oils at the diagnostic doses tested, except for $C$. tinctorium and S. longepedunculata, for which resistance was suspected because the mortality was less than $97 \%$. Then, according to the WHO protocols [78], diagnostic doses are defined as twice the lethal concentration (LC) for $99 \%$ mortality ( $\mathrm{LC}_{99}$ ) on sensitive strains.

According to Bossou et al. [77], C. citratus, E. tereticornis, E. citriodora, C. ambrosioides and C. schoenanthus are potential promising plant sources for alternative compounds to pyrethroids for the control of the Anopheles malaria vector in Benin, where pyrethroid resistance was detected in the south part of the country, due to the massive use of permethrin and DDT. On the contrary, with the topical application, permethrin and deltamethrin seem to be toxic than the EOs in An. gambiae populations tested in a study done by Norris [76].

\section{Repellency Effects of EOs against Anopheles Mosquito Adults}

The repellent ability of EOs seems to be the major promising entomological use in the human health. According to Choochote [79], repellents are substances that act locally or at a distance, deterring an arthropod from flying, landing on or biting humans or animals (or any surface in general). It is also defined as a phenomenon that prevents a pest's ability to track, locate and/or recognize its host. Hence, a repellent phenomenon can be a movement away from an odor source, but also an inability to find the host [80]. Recently, a review on repellency effects on diseases vectors has been published [25]. This review reported current evaluation approach of EOs and various methodology of repellents effects of synergists of EOs constituents.

In terms of methodology, the human bait method is mainly used as a common procedure. However, studies including positive controls are very scarce. In 2016, the review of Deletre et al. [80] identified five (5) types of repellent on the basis of the observed insect behavior: (i) true repellent (also called expellent, spatial repellent), which corresponds to an oriented movement of the insect away from an odor source without direct contact, (ii) odor masking (also called attraction inhibition), which is either a reduction in the attractiveness of the host or a disruption of the localization of the host by the odor cue, (iii) contact irritancy (also called landing inhibition or excito-repellent), an oriented movement of the insect away from a chemical after direct contact, (iv) deterrence (also called antifeeding, suppressant, anorexigenic and anti-appetant), which corresponds to a disruption of feeding activity by contact with or ingestion of a chemical, and (v) visual masking, which defines a reduction in the attractiveness of the host or a disruption of the localization of the host by a visual cue. 
Table 4. Essential oils' toxicity against Anopheles sp. adults.

\begin{tabular}{|c|c|c|c|c|c|c|c|c|c|}
\hline Plant Species & Plant Organ & Extraction Method & Mosquito & Methods & $\mathrm{LC}_{50}(\mathrm{mg} / \mathrm{L})$ & $\mathrm{LD}_{50}$ ( $\mu \mathrm{g} / \mathrm{g}$ Mosquito) & $\mathrm{LC}_{95}$ (mg/mat) & $\mathrm{LC}_{99}(\mathrm{mg} / \mathrm{L})$ & Ref. \\
\hline${ }^{*}$ Deltamethrin & & & An. gambiae & TA & & 0.003 & & & [76] \\
\hline${ }^{*}$ Permethrin & & & An. gambiae & TA & & 0.6 & & & [76] \\
\hline Apium graveolens $\mathrm{L}$. & NI & NI & An. gambiae & TA & & 600.0 & & & [76] \\
\hline Apium graveolens $\mathrm{L}$. & NI & NI & An. gambiae & TA & & 4.5 & & & [76] \\
\hline Apium graveolens $\mathrm{L}$. & seed & NI & An. gambiae & TA & & 6.6 & & & [76] \\
\hline Artemisia absinthium & NI & NI & An. gambia & TA & & 12.0 & & & [76] \\
\hline Cedrus sp. & NI & NI & An. gambiae & TA & & 3.8 & & & [76] \\
\hline Chenopodium ambrosioides & Leafy stems & Hydrod. & An. gambiae & WHOTK & 0.9 & & & 2.1 & [77] \\
\hline Cinnamoтum zeylanicum & Bark & Hydrod. & An. stephensi & MA & & & 286.2 & & [36] \\
\hline Cinnamoтun zeylanicum & NI & NI & An. gambiae & TA & & 2.9 & & & [76] \\
\hline Citrus sinensis & NI & NI & An. gambiae & TA & & 11.1 & & & [76] \\
\hline Cochlospermum planchonii & Root & Hydrod. & An. gambiae & WHOTK & 2.3 & & & 7.6 & [77] \\
\hline Cuminum сутіпum & Seed & Hydrod. & An. stephensi & MA & & & 305.2 & & [36] \\
\hline Curcuma longa & Rhizome & Hydrod. & An. stephensi & MA & & & 302.6 & & [36] \\
\hline Cymbopogon citratus & Leaf & Hydrod. & An. gambiae & WHO test kits & 0.2 & & & 0.4 & [77] \\
\hline Cymbopogon citratus & NI & NI & An. gambiae & TA & & 3 & & & [76] \\
\hline Cymbopogon schoenanthus & Leafy stems & Hydrod. & An. gambiae & WHOTK & 1.57 & & & 2.7 & [77] \\
\hline Cymbopogon winterianus & NI & NI & An. gambiae & TA & & 3.9 & & & [76] \\
\hline Eucalyptus tereticornis & Leaf & Hydrod. & An. gambiae & WHOTK & 0.148 & & & 1.4 & [77] \\
\hline Gaultheria procumbens & NI & NI & An. gambiae & TA & & 11.1 & & & [76] \\
\hline Litsea cubeba & NI & NI & An. gambiae & TA & & 4.0 & & & [76] \\
\hline Mentha piperita & NI & NI & An. gambiae & TA & & 6.8 & & & [76] \\
\hline Myristica fragrans & NI & NI & An. gambiae & TA & & 10.5 & & & [76] \\
\hline Ocimum basilicum & Leaf & Hydrod. & An. stephensi & MA & & & 316.4 & & [36] \\
\hline Origanum vulgare & NI & NI & An. gambiae & TA & & 1.6 & & & [76] \\
\hline Pelargonium graveolens & NI & NI & An. gambiae & TA & & 2.6 & & & [76] \\
\hline Petroselinum crispum & NI & NI & An. gambiae & TA & & 5.0 & & & [76] \\
\hline Pimpinella anisum & Seed & Hydrod. & An. stephensi & MA & & & 378.5 & & [36] \\
\hline Piper nigrum & NI & NI & An. gambiae & TA & & 8 & & & [76] \\
\hline Rosmarinus officinalis & NI & NI & An. gambiae & TA & & 31 & & & [76] \\
\hline Rosmarinus officinalis & Shoot & Hydrod. & An. stephensi & MA & & & 282.6 & & [36] \\
\hline Sassafras sp. & NI & NI & An. gambiae & TA & & 10 & & & [76] \\
\hline Sesamum indicum & NI & NI & An. gambiae & TA & & 5.9 & & & [76] \\
\hline Syzygium aromaticum & leaf & NI & An. gambiae & TA & & 1.5 & & & [76] \\
\hline
\end{tabular}

* Chemical Positive controls; NI: Information is not available; TA = Topical application; MA = Mat machine, WHOTK = WHO test kits, $\mathrm{LC}_{50}$, $\mathrm{LC}_{95}$ and $\mathrm{LC}_{99}$ were Lethal Concentration that killed 50\%, $95 \%$ and $99 \%$ adults mosquitoes $\mathrm{LC}_{50}, \mathrm{LC}_{95}$ and $\mathrm{LC}_{99}$ were Lethal Concentration that killed $50 \%$, $95 \%$ and $99 \%$ adults mosquitoes $\mathrm{LC}_{50}$, $\mathrm{LC}_{95}$ and $\mathrm{LC}_{99}$ were $\mathrm{Lethal}$ Concentration that killed 50\%, 95\% and 99\% adults mosquitoes, LD 50 was Lethal Dose that killed $50 \%$ of adults mosquitoes. 
Amer and Mehlhorn [81] tested essential oils from 41 plants against An. stephensi using the human-bait technique [82]. The twelve most effective oils were those of Nepeta cataria, Jasminum grandiflorum, Cymbopogon citratus, Cinnamomum zeylanicum, Melaleuca leucadendron, Amyris balsamifera, Melaleuca quinquenervia, Tagetes minuta, Viola odorata, Santalum album, Litsea cubeba and Ferula galbaniflua which induced a maximum protection time of $8 \mathrm{~h}$ and showed repellency percentage of $100 \%$ as that of DEET (The positive control). By using the same technique, Rajkumar and Jebanesan [83] evaluated EO of Centella asiatica L., Ipomoea cairica L., Momordica charantia L., Psidium guajava L. and Tridax procumbens L. against An. stephensi. The EOs of I. cairica, M. charantia and T. procumbens at $6 \%$ concentration exhibited relatively high repellency effect $(>5 \mathrm{~h})$, followed by C. asiatica and P. guajava which showed less effective $(<2.5 \mathrm{~h})$ [83]. Phasomkusolsil and Soonwera [84], tested the essential oils of Cymbopogon citratus, Cymbopogon nardus, Cananga odorata, Citrus sinensis, Eucalyptus citriodora, Ocimum basilicum and Syzygium aromaticum against Anopheles dirus at $0.21 \mathrm{mg} / \mathrm{cm}^{2}$. The repellency percentages were $84 \%$ to $98 \%$ and protection time varied from 24 to $132 \mathrm{~min}$ (Table 5).

In addition to their high toxicity against Anopheles sp. adults, the EOs from Cymbopogon winterianus, Cuminum cyminum, Cinnamomum zeylanicum and Thymus vulgaris possessed repellency and irritant actions by using treated paper technology recommended by the WHO [39]. In the repellent tests and at $1 \%$, the proportion of mosquitoes escaping was above those found when permethrin and DEET was used as the positive controls [39]. Also, these EOs were irritants as well as DEET and permethrin (positive controls).

Abagli and Alavo [85] showed the potential of H. suaveolens EO as a mosquito repellent by the human-bait technique. After $6 \mathrm{~h}$ application, the mean number of An. gambiae that landed on treated volunteers was 0.50 and 0.45 for $10 \% \mathrm{H}$. suaveolens essential oil and DEET respectively, against six mosquitoes for the control subjects. H. suaveolens essential oil and DEET possess similarly effects.

\section{Biologically Active Components of Essential Oils}

The insecticidal activity of EOs is dependent on their chemical composition and on interactions between individual compounds [86]. Studies reported that EO activity is the result of their inherent biologically active components (Tables 3 and 6). Generally, EO showing higher efficiency against larvae and adults mosquitoes was dominated by monoterpenes (Table 6).

The larvicidal activities of the essential oil can be related to its main compounds. The three main components of the essential oil of Mentha spicata-carvone, cis-carveol, and limonene-were responsible of its larvicidal activity against An. Stephensi [87]. Also, $\alpha$-humulene and $\beta$-elemene, two main components of the essential oil of Syzygium zeylanicum, appeared highly effective against An. subpictus with $\mathrm{LC}_{50}$ values of 6.19 and $10.26 \mu \mathrm{g} / \mathrm{mL}$, respectively against $83.11 \mu \mathrm{g} / \mathrm{mL}$ for the whole essential oil [65]. Ar-turmerone, a major component of Curcuma longa leaf and rhizome essential oils showed higher and lower efficiency against larvae of An. quadrimaculatus than that of the whole oil from Curcuma longa rhizome and leaf, respectively [50].

Carvacrol and terpinen-4-ol, two major constituents extracted from the Ocimum vulgare EO appeared to be most effective against An. stephensi ( $\mathrm{LC}_{50}=21.15$ and $43.27 \mu \mathrm{g} / \mathrm{mL}$, respectively) and An. subpictus larvae (LC $=24.06$ and $47.73 \mu \mathrm{g} / \mathrm{mL}$, respectively) [88]. The three main components of the essential oil of Plectranthus Barbatus-eugenol, $\alpha$-pinene and $\beta$-caryophyllene-exerted the most potent larvicidal activity against $A n$. subpictus $\left(\mathrm{LC}_{50}=25.45,32.09\right.$ and $41.66 \mu \mathrm{g} / \mathrm{mL}$, respectively) [63]. 
Table 5. Plant essential oils with high repellency to Anopheles species.

\begin{tabular}{|c|c|c|c|c|c|c|c|c|c|}
\hline Plant Species & Plant Organ & $\begin{array}{l}\text { Extraction } \\
\text { Method }\end{array}$ & Moquito & Test Method & $\begin{array}{l}\text { Concentration } \\
\left(\mathrm{mg} / \mathrm{cm}^{2}\right)\end{array}$ & $\begin{array}{l}\text { Protection } \\
\text { Time (mn) }\end{array}$ & $\%$ Repellency & $\begin{array}{c}\mathrm{RD}_{95} \\
\text { (mg/mat) }\end{array}$ & Ref \\
\hline Amyris balsamifera & NI & NI & An. stephensi & Human bait & 0.5 & 480.0 & 100.0 & & [81] \\
\hline Anethum graveolens & NI & NI & An. stephensi & Human bait & 0.5 & 210.0 & 71.4 & & [81] \\
\hline Aniba rosaeodora & NI & NI & An. stephensi & Human bait & 0.5 & 390.0 & 4.8 & & [81] \\
\hline Anthemis nobilis & NI & NI & An. stephensi & Human bait & 0.5 & 480.0 & 76.2 & & [81] \\
\hline Boswellia carteri & & & An. stephensi & Human bait & 0.5 & 300.0 & 19.0 & & [81] \\
\hline Cananga odorata & Flower & Steamd. & An. dirus & Human bait & 0.2 & 24.0 & 92.0 & & [84] \\
\hline Chamaemelum nobile & NI & NI & An. stephensi & Human bait & 0.5 & 330.0 & 47.6 & & [81] \\
\hline Cinnamomum camphora & NI & NI & An. stephensi & Human bait & 0.5 & 480.0 & 42.8 & & [81] \\
\hline Cinnamomum zeylanicum & NI & NI & An. stephensi & Human bait & 0.5 & 480.0 & 100.0 & & [81] \\
\hline Cinnamomum zeylanicum & Bark & Hydrod. & An. stephensi & Cage test & & & & 49.6 & [36] \\
\hline Citrus limon & NI & NI & An. stephensi & Human bait & 0.5 & 420.0 & 9.5 & & [81] \\
\hline Citrus sinensis & Fruit & Steamd. & An. dirus & Human bait & 0.21 & 24 & 84 & & [84] \\
\hline Curcuma longa & Rhizome & Hydrod. & An. stephensi & Cage test & & & & 93.7 & [36] \\
\hline Cymbopogon citratus & Leaf and stem & Steamd. & An. dirus & Human bait & 0.2 & 132.0 & 98.0 & & [84] \\
\hline Cymbopogon citratus & NI & NI & An. stephensi & Human bait & 0.5 & 480.0 & 100.0 & & [81] \\
\hline Cymbopogon nardus & Leaf & Steamd. & An. dirus & Human bait & 0.2 & 90.0 & 98.0 & & [84] \\
\hline Cymbopogon winterianus & NI & NI & An. stephensi & Human bait & 0.5 & 480.0 & 52.4 & & [81] \\
\hline DEET & NI & NI & An. stephensi & Human bait & 0.5 & 480.0 & 100.0 & & [81] \\
\hline Eucalyptus citriodora & Leaf & Steamd. & An. dirus & Human bait & 0.2 & 30.0 & 86.0 & & [84] \\
\hline Eucalyptus citriodora & NI & NI & An. stephensi & Human bait & 0.5 & 480.0 & 52.4 & & [81] \\
\hline Eucalyptus dives & NI & NI & An. stephensi & Human bait & 0.5 & 480.0 & 38.1 & & [81] \\
\hline Eucalyptus globulus & NI & NI & An. stephensi & Human bait & 0.5 & 330.0 & 28.6 & & [81] \\
\hline Eucalyptus radiata & NI & NI & An. stephensi & Human bait & 0.5 & 480.0 & 42.8 & & [81] \\
\hline Ferula galbaniflua & NI & NI & An. stephensi & Human bait & 0.5 & 480.0 & 100.0 & & [81] \\
\hline Glycina max & NI & NI & An. stephensi & Human bait & 0.5 & 480.0 & 76.2 & & [81] \\
\hline Glycina soja & NI & NI & An. stephensi & Human bait & 0.5 & 480.0 & 9.5 & & [81] \\
\hline Helichrysum italicum & NI & NI & An. stephensi & Human bait & 0.5 & 360.0 & 47.6 & & [81] \\
\hline Jasminum grandiflorum & NI & NI & An. stephensi & Human bait & 0.5 & 480.0 & 100.0 & & [81] \\
\hline Juniperus communis & NI & NI & An. stephensi & Human bait & 0.5 & 480.0 & 76.2 & & [81] \\
\hline Juniperus virginiana & NI & NI & An. stephensi & Human bait & 0.5 & 480.0 & 38.1 & & [81] \\
\hline Lavandula angustifolia & NI & NI & An. stephensi & Human bait & 0.5 & 480.0 & 80.9 & & [81] \\
\hline Lippia citriodora & NI & NI & An. stephensi & Human bait & 0.5 & 330.0 & 38.1 & & [81] \\
\hline Litsea cubeba & NI & NI & An. stephensi & Human bait & 0.5 & 480.0 & 100.0 & & [81] \\
\hline Melaleuca leucadendron & NI & NI & An. stephensi & Human bait & 0.5 & 480.0 & 100.0 & & [81] \\
\hline Melaleuca quinquenervia & NI & $\mathrm{NI}$ & An. stephensi & Human bait & 0.5 & 480.0 & 100.0 & & [81] \\
\hline
\end{tabular}


Table 5. Cont.

\begin{tabular}{|c|c|c|c|c|c|c|c|c|c|}
\hline Plant Species & Plant Organ & $\begin{array}{c}\text { Extraction } \\
\text { Method }\end{array}$ & Moquito & Test Method & $\begin{array}{c}\text { Concentration } \\
\left(\mathrm{mg} / \mathrm{cm}^{2}\right)\end{array}$ & $\begin{array}{l}\text { Protection } \\
\text { Time (mn) }\end{array}$ & \% Repellency & $\begin{array}{c}\mathrm{RD}_{95} \\
\text { (mg/mat) }\end{array}$ & Ref. \\
\hline Mentha piperita & NI & NI & An. stephensi & Human bait & 0.5 & 390.0 & 57.1 & & {$[81]$} \\
\hline Myrtus communis & NI & NI & An. stephensi & Human bait & 0.5 & 390.0 & 42.8 & & [81] \\
\hline Nepeta cataria & NI & NI & An. stephensi & Human bait & 0.5 & 480.0 & 100.0 & & [81] \\
\hline Ocimum basilicum & Leaf & Hydrod. & An. stephensi & Cage test & & & & 75.0 & [36] \\
\hline Ocimum basilicum & Leaf & Steamd. & An. dirus & Human bait & 0.2 & 96.0 & 96.0 & & [84] \\
\hline Ocimum basilicum & NI & NI & An. stephensi & Human bait & 0.5 & 210.0 & 66.7 & & [81] \\
\hline Olea europaea & NI & NI & An. stephensi & Human bait & 0.5 & 480.0 & 71.4 & & [81] \\
\hline Pelargonium graveolens & NI & NI & An. stephensi & Human bait & 0.5 & 480.0 & 61.9 & & [81] \\
\hline Picea excelsa & NI & NI & An. stephensi & Human bait & 0.5 & 180.0 & 19.0 & & [81] \\
\hline Pimpinella anisum & Seed & Hydrod. & An. stephensi & Cage test & & & & 154.1 & [36] \\
\hline Piper nigrum & NI & NI & An. stephensi & Human bait & 0.5 & 180.0 & 61.9 & & [81] \\
\hline Rosmarinus officinalis & Shoot & Hydrod. & An. stephensi & Cage test & & & & 38.9 & [36] \\
\hline Rosmarinus officinalis & NI & $\mathrm{NI}$ & An. stephensi & Human bait & 0.5 & 480.0 & 100.0 & & [81] \\
\hline Salvia sclarea & NI & NI & An. stephensi & Human bait & 0.5 & 300.0 & 19.0 & & [81] \\
\hline Santalum album & NI & NI & An. stephensi & Human bait & 0.5 & 480.0 & 100.0 & & [81] \\
\hline Syzygium aromaticum & Flower & Steamd. & An. dirus & Human bait & 0.2 & 96.0 & 98.0 & & [84] \\
\hline Tagetes minuta & NI & NI & An. stephensi & Human bait & 0.5 & 480.0 & 100.0 & & [81] \\
\hline Thymus serpyllum & NI & NI & An. stephensi & Human bait & 0.5 & 450.0 & 33.3 & & [81] \\
\hline Viola odorata & NI & NI & An. stephensi & Human bait & 0.5 & 480.0 & 100.0 & & [81] \\
\hline
\end{tabular}

DEET: $N, N$-diethyl-3-methylbenzanmide, used as repellent positive control; $\mathrm{RD}_{50}$ and $\mathrm{RD}_{95}$ are the doses that repel $50 \%$ and $95 \%$ of adults, respectively [44]. 
Table 6. Essential oil composition and their activity against Anophelineae populations.

\begin{tabular}{|c|c|c|c|c|c|c|c|c|c|}
\hline Plant Species & Essential Oil Major Components (\%) & $\begin{array}{l}\text { Mono } \\
\text { Hydro }\end{array}$ & $\begin{array}{c}\text { Mono } \\
\text { Oxy }\end{array}$ & $\begin{array}{l}\text { Sesqui } \\
\text { Hydro }\end{array}$ & $\begin{array}{c}\text { Sesqui } \\
\text { Oxy }\end{array}$ & Mosquito Species & $\begin{array}{c}\text { Larvicidal } \\
\mathrm{LC}_{50}(\mathrm{mg} / \mathrm{L})\end{array}$ & $\begin{array}{c}\text { Adulcidal } \\
\mathrm{LC}_{50}(\mathrm{mg} / \mathrm{L})\end{array}$ & Ref. \\
\hline Ocimum basilicum & Linalool (52.4), Methyl eugenol (18.7) & 9.9 & 79.8 & 13.4 & & An. subpictus & 9.7 & & [56] \\
\hline Blumea densiflora & $\begin{array}{l}\text { Borneol (11.4), germacrene D. (8.66), } \\
\beta \text {-caryophyllene (6.6), } \gamma \text {-terpinene (4.3), sabinene } \\
\text { (4.3), and } \beta \text {-bisabolene (4.2) }\end{array}$ & 25.9 & 25.2 & 29.3 & 10.7 & An. anthropophagus & 10.0 & & [42] \\
\hline Tagetes patula & $\begin{array}{l}\text { Limonene (13.60), terpinolene (11.2), Z- } \beta \text {-ocimene } \\
\text { (8.3), E-caryophyllene ( } 8.0) \text {, piperitone (6.1), } \\
p \text {-cymen-8-ol (5.4), piperitenone (4.9) }\end{array}$ & 40.6 & 21.2 & 9.3 & 3.1 & An. stephensi & 12.1 & & [66] \\
\hline Ferulago carduchorum & $\begin{array}{l}(Z) \text { - } \beta \text {-ocimene (43.3), } \alpha \text {-pinene (18.2), bornyl } \\
\text { acetate (3.9) }\end{array}$ & 78.6 & 5.9 & 5.9 & 0.7 & An. stephensi & 12.8 & & [53] \\
\hline Chloroxylon swietenia & $\begin{array}{l}\text { Geijerene (26.9), limonene (15.2), germacrene D } \\
\text { (10.6), pregeijerene ( } 7.8)\end{array}$ & 22.9 & 2.7 & 53.9 & 3.0 & An. stephensi & 14.9 & & [77] \\
\hline Feronia limonia & $\begin{array}{l}\text { Estragole (34.7), } \beta \text {-pinene (23.6), (Z)-caryophyllene } \\
\text { (11.0), methyl (Z)-caryophyllene (11.0). }\end{array}$ & 32.5 & 46.9 & 13.0 & 7.2 & An. Stephensi & 15.0 & & [52] \\
\hline Cymbopogon citratus & $\begin{array}{l}\text { Geranial (39.3), neral (21.9), geraniol (15.6), } \\
\text { myrcene (14.00) }\end{array}$ & 15.8 & 81.6 & 0.5 & & An. gambiae & 18.0 & & [38] \\
\hline Chloroxylon swietenia & $\begin{array}{l}\text { Limonene (12.9), geijerene (17.7), pregeijerene } \\
(9.92) \text {, germacrene D (8.84) }\end{array}$ & 21.1 & 5.47 & 41.5 & 8.6 & An. stephensi & 19.0 & & [45] \\
\hline Bunium persicum & $\begin{array}{l}p \text {-Cuminaldehyde (23.5), } \\
\alpha \text {-methylbenzenemethanol (14.6), camphor (13.5), } \\
\gamma \text {-terpinene (13.1), } \beta \text {-cymene (8.5) }\end{array}$ & 33.3 & 53.7 & 5.8 & 1.5 & An. stephensi & 27.7 & & [43] \\
\hline Plectranthus amboinicus & $\begin{array}{l}\text { Carvacrol (28.6), thymol (21.7), } \alpha \text {-humulene (9.7), } \\
\text { undecanal (8.3) }\end{array}$ & 15.1 & 64.2 & 11.7 & 8.6 & An. stephensi & 28.4 & & [62] \\
\hline Citrus aurantium & D,L-limonene(94.8) & 97.4 & 0.7 & 0.1 & 0.06 & A. stephensi & 31.2 & & [47] \\
\hline Plectranthus mollis & $\begin{array}{l}\text { Piperitone oxide (23.7), fenchone (19.2), } \\
\text { piperitenone oxide (13.0), } \beta \text {-caryophyllene (10.3) }\end{array}$ & 5.6 & 60.2 & 18.3 & 0.9 & An. stephensi & 33.5 & & [55] \\
\hline Cymbopogon citratus & $\begin{array}{l}\text { Myrcene (11.4), neral (30.2), geranial (32.8) } \\
\delta \text {-Cadinene (16.8), } \beta \text {-pinene (7.2), } \beta \text {-bisabolene (5.6), } \\
\alpha \text {-phellandrene (4.7), myristicin (4.), } \alpha \text {-pinene (3.9), }\end{array}$ & 14.1 & 74.5 & 1.3 & 0.1 & An. funestus & 34.6 & & [51] \\
\hline Piper capense & $\begin{array}{l}\text { sabinene (3.8), } \beta \text {-cubebene (3.3), bicyclogermacrene } \\
\text { (3.3), limonene (3.1), } \beta \text {-phellandrene (2.5), linalool } \\
\text { (2.4), spathulenol (2.4) }\end{array}$ & 30.6 & 4.3 & 43.9 & 6.1 & An. gambiae & 34.9 & & [60] \\
\hline Zingiber nimmoni & $\begin{array}{l}\beta \text {-Caryophyllene (26.9), } \alpha \text {-humulene (19.6), } \\
\alpha \text {-cadinol (5.20), myrcene (5.10) }\end{array}$ & 19.7 & 9.5 & 51.9 & 16.2 & An. stephensi & 41.2 & & [59] \\
\hline Amomum subulatum & $\begin{array}{l}\text { 1.8-cineole (39.8), } \alpha \text {-terpineol (11.5), } \beta \text {-pinene (4.2), } \\
\text { terpinen-4-ol (3.9) }\end{array}$ & 50.7 & 23.7 & 9.0 & 11.0 & An. subpictus & 41.2 & & [40] \\
\hline Mentha spicata & carvone (48.6), cis-carveol (21.3), limonene (11.3) & 11.3 & 83.2 & 3.2 & 0.7 & An. stephensi & 49.7 & & [87] \\
\hline Artemisia gilvescens & $\begin{array}{l}\text { Camphor (13.5), eucalyptol (12.1), terpine-4-ol (9.6), } \\
\text { germacrene D (8.6) }\end{array}$ & 10.1 & 53.9 & 18.9 & 9.0 & An. anthropophagus & 49.9 & & [42] \\
\hline Plectranthus amboinicus & $\begin{array}{l}\text { Carvacrol (29.2), thymol (21.7), } \alpha \text {-humulene (9.7), } \\
\text { undecanal (8.3) }\end{array}$ & 14.9 & 64.2 & 9.7 & 7.9 & An. gambiae & 55.2 & & [61] \\
\hline Murraya exotica & $\beta$-Humulene (40.6), benzyl benzoate (23.9) & 5.66 & 1.0 & 61.5 & 6.01 & An. stephensi & 56.3 & & [58] \\
\hline
\end{tabular}


Table 6. Cont.

\begin{tabular}{|c|c|c|c|c|c|c|c|c|c|}
\hline Plant Species & Essential Oil Major Components (\%) & $\begin{array}{l}\text { Mono } \\
\text { Hydro }\end{array}$ & $\begin{array}{c}\text { Mono } \\
\text { Oxy }\end{array}$ & $\begin{array}{l}\text { Sesqui } \\
\text { Hydro }\end{array}$ & $\begin{array}{c}\text { Sesqui } \\
\text { Oxy }\end{array}$ & Mosquito Species & $\begin{array}{c}\text { Larvicidal } \\
\mathrm{LC}_{50}(\mathrm{mg} / \mathrm{L})\end{array}$ & $\begin{array}{c}\text { Adulcidal } \\
\mathrm{LC}_{50}(\mathrm{mg} / \mathrm{L})\end{array}$ & Ref. \\
\hline Zanthoxylum armatum & $\begin{array}{l}\text { Linalool (57.0), limonene (19.8), E-methyl } \\
\text { cinnamate (5.7) }\end{array}$ & 21.7 & 75.0 & & & An. stephensi & 58.0 & & [69] \\
\hline Coleus aromaticus & thymol (82.6) & 2.8 & 88.7 & 3.2 & 1.3 & An. subpictus & 60.3 & & [48] \\
\hline Zhumeria majdae & Linalool (31.2), camphor (38.5) & 18.9 & 77.4 & 0.6 & 0.7 & An. stephensi & 61.3 & & [70] \\
\hline Lavandula gibsoni & Thymol (10.4), $\alpha$-terpinolene (22.2) & 5.6 & 60.2 & 18.3 & 0.9 & An. stephensi & 62.8 & & [55] \\
\hline Origanum vulgare & Carvacrol (38.3), terpiene-4-ol (28.7) & 7.7 & 80.6 & 5.2 & 3.7 & An. stephensi & 67.0 & & [88] \\
\hline Cryptomeria japonica & Kau-16-rene (23.3), $\beta$-elemol (18.3) & 28.9 & 6.5 & 0.4 & 39.0 & An. gambiae & 67.1 & & [49] \\
\hline Origanum vulgare & Carvacrol (38.30), terpiene-4-ol (28.7) & 7.7 & 80.6 & 5.2 & 3.7 & An. subpictus & 74.1 & & [88] \\
\hline Trachyspermuт атті & Thymol (66.7), $p$-cymene (17.4), $\gamma$-terpenene (10.1) & 29.8 & 67.9 & & & An. stephensi & 80.8 & & [68] \\
\hline Mentha spicata & $\begin{array}{l}\text { Piperitenone oxide (71.1), carvone (5.8), } \\
\beta \text {-caryophyllene (2.3), limonene (1.3) }\end{array}$ & 1.4 & 76.9 & 2.3 & & An. stephensi & 82.9 & & {$[57]$} \\
\hline Syzygium zeylanicum & $\alpha$-Humulene (37.8), $\beta$-elemene (10.7) & 1.2 & 0.9 & 64.6 & 24.9 & An. subpictus & 83.1 & & [65] \\
\hline Plectranthus barbatus & $\begin{array}{l}\text { Eugenol (31.1), } \alpha \text {-pinene (19.4), } \beta \text {-caryophyllene } \\
\text { (18.4). }\end{array}$ & 25.2 & 31.1 & 38.9 & 2.6 & An. subpictus & 84.2 & & [89] \\
\hline Ocimum canum & 1.8-Cineole (29.4), linalool (19.1), Perpinen-4-ol (7.5) & 10.4 & 35.2 & 8.4 & & An. funestus & 91.2 & & [51] \\
\hline Eucalyptus tereticornis & $\begin{array}{l}\text { p-Cymene (16.7), caryophyllene oxide (14.2), } \\
\text { spathulenol (13.5). cryptone (11.4) }\end{array}$ & 23.9 & 20.0 & 2.0 & 34.7 & An. gambiae & & 0.1 & [77] \\
\hline Cymbopogon citratus & Neral (33.1), geranial (44.3) & 12.9 & 81.1 & 0.2 & 0.5 & An. gambiae & & 0.2 & [77] \\
\hline $\begin{array}{l}\text { Chenopodium } \\
\text { ambrosioides }\end{array}$ & $\begin{array}{l}\text { Ascaridole (41.9), } \alpha \text {-terpinene (16.5), } p \text {-cymene } \\
\text { (14.4), isoascridole (7.5) }\end{array}$ & 31.6 & 51.5 & & & An.gambiae & & 1.0 & [44] \\
\hline $\begin{array}{l}\text { Cymbopogon } \\
\text { schoenanthus }\end{array}$ & piperitone (58.9), $\delta$-2-carene (15.5) & 19.8 & 63.5 & 2.3 & 12.8 & An. gambiae & & 1.6 & [77] \\
\hline
\end{tabular}

Mono hydro: Monoterpenes hydrocarbon; Mono Oxy: Monoterpenes Oxygenated; Sesquiterpenes hydro: Sesquiterpenes hydrocarbon; Sesquiterpenes Hydro; Sesquiterpenes Oxygenated. 
The minor compounds could be also implicated in the adult mosquito toxicity of an essential oil. Thus, the toxicity of C. winterianus and C. cymimum oils could be due to their minor compounds, whereas this was not the case for C. zeylanicum and T. vulgaris oils, which toxicity could be due to cinnamaldehyde and the major compounds in the blend [90]. $\alpha$-Pinene, limonene, terpinolene, citronellol, citronellal, camphor and thymol are common constituents of a number of essential oils that show mosquito repellent activity [91-95]. Among the sesquiterpenes, $\beta$-caryophyllene is considered to be a strong repellent against Anopheles sp. [91,96,97]. Phytol, a linear diterpene alcohol, possessed high repellent activity against An. gambiae in Kenya [98]. Repellent and irritant effects of Thymus vulgaris, Cuminum cymimum and Cinnamomum zeylanicum essential oils are usually considered due to one major compound [90]. Thus, the repellency effects of T. vulgaris, Cymbopogon winterianus and Cuminum cymimum was attributed to their main components carvacrol, citronellal, geraniol and cuminaldehyde, respectively [90]. Moreover, thymol and/or carvacrol; citronellal, geraniol, and/or citronellol; cuminaldehyde; and cinnamaldehyde, respectively, are implicated in T. vulgaris, C. winterianus, C. cymimum and C. zeylanicum oils' irritant effects. In fact, there was no significant difference between the single compounds and associated essential oils [90].

\section{Synergistic and Antagonistic Phenomena}

The inherent activity of an EO can be expected to be related to the chemical configuration of the components, the proportions in which they are present and to interactions between them [99]. An additive effect is observed when the combined effects are equal to the sum of the individual effects. Antagonism is observed when the effects of one or both compounds are less when they are applied together than when applied alone. Synergism is observed when the effect of the combined substances is greater than the sum of the individual effects [100]. In some cases, the whole essential oil exhibited a greater pesticidal activity than its major components isolated, suggesting that the minor components are critical to the activity and may have a synergistic effect or potentiating influence [101].

In general, it appears that the effect of an active compound could be enhanced by other major compounds and/or modulated by minor compounds to give additive or synergistic effects. For example, repellency and toxicity of $(E)$-cinnamaldehyde could be synergistically enhanced by minor compounds like benzaldehyde, coumarin, phenyl ethyl alcohol, and ( $Z$ )-cinnamaldehyde, while the irritancy of carvacrol appeared to be reduced by minor compounds such as myrcene, borneol, $\alpha$-pinene, $\gamma$-terpinene, terpinen-4-ol, limonene, and $\alpha$-thujene [90]. Accordingly, minor constituents found in low percentages may act as synergists, enhancing the effectiveness of the major constituents through a variety of mechanisms [102]. A synergistic phenomenon among the metabolites may result in a higher bioactivity compared to the isolated components [97]. This synergistic effect is also observed with mixtures of oils. The high insect repellency and toxicity of the mixed oils might have resulted from synergistic action of the main components in the oils.

Studies on the interactions between oil compounds against An. gambiae remain very scarce. Recently, Deletre [90] investigated and showed a complexity in the biological activity of EOs for toxicity, irritancy and repellent tests. Nevertheless, no correlation was found between the activity of EO and activity from their major components. Many of the repellent studies have shown that synthetic compounds or blends of pure compounds are less effective when compared to the activity of their corresponding essential oils [25]. That was the case of cinnamaldehyde, the major constituent of C. zeylanicum [90].

In terms of toxicity, no synergistic effect was observed between the oil components but an antagonist effect was observed between a Cinnamaldehyde, a major constituent of C. zelanicum and other components explaining their low value [90]. An antagonistic effect between cuminaldehyde (a major component) and another constituent will be suspected for Cuminum cyminum EO due to the fact that the cumin blend was less irritant than essential oil. 


\section{Mechanisms of Action of Essential Oil Components}

The modes of action of EOs are well documented [103], but nevertheless this review will highlight the target sites of EOs. Most monoterpenes are toxic to insects by penetrating the body through the respiratory system (fumigant effect), the cuticle (contact effect) or through the digestive system in case of ingestion [104,105].Several monoterpenes present in EOs are neurotoxic to insects. Rattan [106] described three major targets of insect neurosystems (the cholinergic, octopamenergic and GABA systems).

Acetylcholinesterase (AChE) plays a key role in cholinergic synapses that are essential for insects and higher animals [107]. It is known to be a class of enzymes that catalyzes the hydrolysis of the neurotransmitting agent acetylcholine (ACh). Inhibition of AChE causes accumulation of acetylcholine in the synapses, so that the post-synaptic membrane is in a state of permanent stimulation, which results in ataxia i.e., general lack of co-ordination in the neuromuscular system, and eventual death $[103,108]$. A number of monoterpenes also act on acetylcholinesterase $[103,109]$. For example, linalool a monoterpene that is the major compound of $C$. sativum essential oil, showed a toxic effect to mosquitoes and was identified as an inhibitor of acetylcholinesterase [105].

According to Abdelgaleil et al. [110] monoterpenes such as cuminaldehyde, 1,8-cineole, limonene and fenchone have strong insecticidal activity and potent AChE inhibitory activity whereas other compounds like geraniol or $R$-carvone have strong insecticidal activity but are weak inhibitors of AChE. Hideyuki and Mitsuo [111] noted that the mixture of monoterpenoids could be active as synergists in the inhibition of AChE. In fact, these authors found out that limonene, linalool and lynalyl acetate mixture showed synergistic effects and were more inhibitory than bergamot oil or single monoterpenoid applications.

Carvacrol, $\alpha$-pinene, and $\beta$-pinene inhibited the activity of Aedes albopictus larvae acetylcholinesterase with IC values of $0.05,0.06$, and $0.19 \mathrm{mg} / \mathrm{mL}$, respectively [112]. Savelev et al. [113] by analysing synergistic and antagonistic interactions of anticholinesterase terpenoids, concluded with the evidence of synergy since the inhibitory activity of individual terpenes was lower than the whole oil. Keane and Ryan [114] mentioned that the validation of an inhibition in vitro of the AChE should be demonstrated additionally by an appropriate effect in vivo. These authors also pointed out the importance of not excluding additional modes of action of monoterpenoids.

Another possible target for essential oil activity is the octopaminergic system of insects. Octopamine is a naturally occurring multifunctional biogenic amine, which plays key roles as a neurotransmitter, neuromodulator and neurohormone in invertebrate systems, with a physiological function similar to that of nornephrine in vertebrates [115]. The acute and sub-lethal behavioral effects of essential oil compounds on insects and other vertebrates are consistent with an octopamenergic target site in insects, which acts by blocking octopamine receptors [116,117].

Physiological functions of OA appears to be mediated by pharmacologically distinct class of octopamine receptors viz. octopamine receptors (myogenic rhythm via Cap concentration), octopamine 2A and octopamine 2B, octopamine 3 (activation of adenylate cyclise activity), which are coupled to different second messenger system belonging to the family of G-protein coupled receptors (G-PCRs) [117-120]. It was subsequently shown that treatment with the octopaminergic antagonist phentolamine effectively inhibited the cyclic AMP levels induced by essential oil treatment, indicating a possible competitive activation of octopaminergic receptors by essential oil constituents.

Enan [117] showed that low doses of eugenol caused a significant increase in adenosine $3^{\prime}, 5$-cyclic monophosphate (cAMP) in the nervous system of P. americana, a similar effect to that of octopamine. The increase in cAMP caused by octopamine was blocked by a mixture of three essential oil constituents: eugenol, $\gamma$-terpineol and cinnamic alcohol. It was also demonstrated that low doses of eugenol significantly decreased octopamine receptor binding.

Ligand-gated chloride channels are recognized to be a target site for insecticides acting as antagonists by stabilizing non-conducting conformations of the chloride channel. Blockage of the 
GABA-gated chloride channel reduces neuronal inhibition, which leads to hyper-excitation of the central nervous system, convulsions, and then death [121].

Based on the $\left[{ }^{3} \mathrm{H}\right]-$-ТВOB binding assay and ${ }^{36} \mathrm{Cl}^{-}$uptake assay, Tong and Coats [122] showed that some monoterpenoids can affect the functioning of insects' GABA systems by binding to the GABA receptor and increasing the chloride uptake activated by GABA. Their studies revealed that in the American cockroach's ventral nerve cord, carvacrol, pulegone, and thymol all significantly increased the ${ }^{36} \mathrm{Cl}^{-}$uptake stimulated by GABA, and that they did not increase the ${ }^{36} \mathrm{Cl}^{-}$uptake in the absence of GABA in the assay system showing that carvacrol, pulegone, and thymol were all positive allosteric modulators for insect's GABA receptor. They modulated the insect GABA system by binding at the receptor and increasing chloride anion influx into the neurons.

Thujone is also classified as a neurotoxic insecticide, which acts on GABA receptors $[123,124]$. Thujone is a competitive inhibitor of $\left[{ }^{3} \mathrm{H}\right]$ EBOB binding (i.e., of the non-competitive blocker site of the GABA-gated chloride channel) and is a reversible modulator of the GABA receptor. It was suggested that thymol potentiates GABA receptors through an unidentified binding site [125].

\section{Microencapsulation and Nanoemulsion Technologies}

Application of essential oils is limited due to their rapid volatility. However, with new currently available technologies, it is possible to improve the duration of action of EOs. Microencapsulation is a technology for packaging an active ingredient in the form of droplets of a solid or liquid material (the core) inside a capsule, that is, a continuous film of polymeric material (the shell) ranging in size from one micron to several millimeters [126].

Microencapsulation processes are categorized into two groups: chemical processes and mechanical or physical processes [126]. The morphology of microcapsules can be as follows: (i) mononuclear containing the shell around the core; (ii) polynuclear that have many cores enclosed within the shell or (iii) matrix with the core material is distributed homogenously in the shell material [126]. Commercial microcapsules usually have a diameter between 3 and $800 \mu \mathrm{m}$ and contain 10-90 wt \% core [127].

The active products of EOs can be encapsulated, used with polymer resins or synergized by other compound like vanillin [105,128]. According to Tawatsin [129], oils from Curcuma longa, Cymbopogon winterianus and Ocimum americanum, especially with the addition of $5 \%$ vanillin, repelled Anopheles dirus under cage conditions for up to eight hours.

The compounds should be selected taking into account to their low toxicity to humans and must provide protection for at least $4 \mathrm{~h}$. According to Deletre [90], compounds from citronella can be potential alternatives to repellents, especially since they are non-toxic individually and when mixed. This explains why this EO is used in microencapsulation experiments. Recently, a simple, low cost, scalable, and reproducible method has been set up by Specos [130]. This technology consists in preparing complex of coacervation microcapsules containing citronella EO. Using this technology, the study aimed at assessing repellent activity by exposure of a human hand and arm covered with treated textiles to Aedes aegypti mosquitoes. According to these authors, fabrics treated with microencapsulated citronella exhibited a higher and longer lasting protection from insects compared to fabrics sprayed with an ethanol solution of the essential oil, assuring a repellent effect higher than $90 \%$ for up to three weeks. Salomon et al. [131] showed that microencapsulation decreased membrane permeation of citronella $\mathrm{EO}$ by at least $50 \%$. Another technology is encapsulated citronella oil nanoemulsion which is prepared by high pressure homogenization at varying amounts of surfactant and glycerol [132]. These authors showed that the release of citronella oil from high amounts of glycerol was much slower than that from the low glycerol amounts resulting in sustained mosquito protection time.

\section{Concluding Remarks}

Pyrethroids are widely used in controlling mosquitoes. They are used in bednet treatments, impregnation of clothes, indoor residual spraying and spatial treatments [133]. The advantage of pyrethroids is their effectiveness at low dosages. They are also toxic, irritant, fast acting, stable and safe 
for humans $[134,135]$. According to Duvallet [133], pyrethroids have four main effects on mosquitoes causing: (i) a spatial repellent effect by deterrence of adults from entering treated rooms; (ii) a contact irritant effect by short-lived settling of mosquitoes on treated bednets or walls; (iii) an anti-feedant effect by inhibition of blood feeding by female mosquitoes and (iv) toxic effect by inducing a knock down (KD) and mortality effect. In Africa, pyrethroid resistance in Anopheles populations is now widespread and could compromise the vector control strategies. Thus, the use of EOs as mosquito control insecticides would be greatly increased if their mode of action is not the same as that of currently used chemical products [136]. This raises the following question: Can EOs be used as an alternative to the pyrethroid resistance?

A number of EOs and several of their individual components exhibit insecticidal activity against several disease vectors. Their mechanism of action depends on component involving several targets such as the cholinergic, octopamenergic and GABA systems [137].

Therefore, knowledge of the physiological mode of action of these active essential oils is of great importance for their future use in health. EOs might be used as alternative to the resistance of pyrethroids if it were demonstrated that only channel sodium voltage dependant target sites are involved in this resistance. In fact, many EOs or their components act as inhibitors of acetylcholinesterase while the sodium channel voltage dependent is mainly implicated in pyrethroid resistance.

However, some cases of resistance to organophosphates and carbamates are reported due to the mutation in acetylcholinesterase gene $1\left(a c e-1^{R}\right)$ [5-7], with a percentage reaching rarely $30 \%$ up till now [138]. Fortunately, this rate may decrease in the absence of OP and carbamate applications. Then, homozygous and heterozygous ace- $1^{R}$ vectors, which survive in the presence of insecticide, may be rapidly outcompeted in the absence of insecticide.

The synergistic interactions between EOs components are interesting; it is possible to mix two or three compounds with different effects to avoid habituation behaviour from mosquitoes. To date, microencapsulation, which improves the duration of action of certain EOs, can favor the use of ingredients of EOs in terms of impregnation of mosquito bednets. In conclusion, EOs have both toxic and repellent actions and could be envisaged as a way for sustainable management of pyrethroid resistance in Africa.

Acknowledgments: Authors are grateful to the project TWAS 15-259 RG/BIO/AF/AC, which supported the study on resistance monitoring. Authors also thank Souleymane BADO from International Atomic Energy Agency (AIEA) and Jean ZIDA for editing this manuscript.

Author Contributions: O.G. and I.N.H.B. conceived and designed the study. O.G. and I.N.H.B. drafted, supervised and reviewed the manuscript. Each author approved the final manuscript.

Conflicts of Interest: The authors declare that they have no competing interests.

\section{Abbreviations}

$\begin{array}{ll}\text { EOs } & \text { Essential Oils } \\ \text { IRS } & \text { Indoor residual spraying } \\ \text { LLINs } & \text { Long-lasting insecticide treated nets } \\ k d r & \text { knock down resistance } \\ \text { LD } & \text { Lethal dose } \\ \text { LC } & \text { Lethal concentration } \\ \text { US EPA } & \text { United States Environmental Protection Agency } \\ \text { WHO } & \text { World Health Organization } \\ \text { DEET } & \text { N,N-diethyl-3-methylbenzamide } \\ \text { AChE } & \text { Acetylcholinesterase } \\ \text { ACh } & \text { Acetylcholine } \\ \text { OA } & \text { Octopamine } \\ \text { GABA } & \text { Gamma aminobutyric acid }\end{array}$




\section{References}

1. WHO. World Malaria Report 2015; World Health Organization: Geneva, Switzerland, 2015.

2. WHO. Global Plan for Insect Management; World Health Organization: Geneva, Switzerland, 2012.

3. Kelly-Hope, L.; Ranson, H.; Hemingway, J. Lessons from the past: Managing insecticide resistance in malaria control and eradication programmes. Lancet Infect. Dis. 2008, 8, 387-389. [CrossRef]

4. Namountougou, M.; Diabaté, A.; Etang, J.; Bass, C.; Sawadogo, S.P.; Gnankinié, O.; Baldet, T.; Martin, T.; Chandre, F.; Simard, F.; et al. First report of the L1014S kdr mutation in wild populations of Anopheles gambiae $\mathrm{M}$ and S molecular forms in Burkina Faso (West Africa). Acta Trop. 2013, 125, 123-127. [CrossRef] [PubMed]

5. Namountougou, M.; Frédéric, S.; Baldet, T.; Diabate, A.; Ouédraogo, J.-B.; Martin, T.; Dabire, R.K. Multiple Insecticide Resistance in Anopheles gambiae s.l. Populations from Burkina Faso, West Africa. PLoS ONE 2012, 7. [CrossRef] [PubMed]

6. Gnankiné, O.; Bassolé, I.H.N.; Chandre, F.; Glitho, I.; Akogbeto, M.; Dabiré, R.K.; Martin, T. Insecticide resistance in Bemisia tabaci Gennadius (Homoptera: Aleyrodidae) and Anopheles gambiae Giles (Diptera: Culicidae) could compromise the sustainability of malaria vector control strategies in West Africa. Acta Trop. 2013, 128, 7-17. [CrossRef] [PubMed]

7. Dabiré, R.; Namountougou, M.; Sawadogo, S.; Yaro, L.; Toé, H.; Ouari, A.; Gouagna, L.-C.; Simard, F.; Chandre, F.; Baldet, T.; et al. Population dynamics of Anopheles gambiae s.l. in Bobo-Dioulasso city: Bionomics, infection rate and susceptibility to insecticides. Parasites Vectors 2012, 5, 127. [CrossRef] [PubMed]

8. Silva, A.P.B.; Santos, J.M.M.; Martins, A.J. Mutations in the voltage-gated sodium channel gene of anophelines and their association with resistance to pyrethroids-A review. Parasites Vectors 2014, 7, 1-14. [CrossRef] [PubMed]

9. Chandre, F.; Darrier, F.; Manga, L.; Akogbeto, M.; Faye, O.; Mouchet, J.; Guillet, P. Status of pyrethroid resistance in Anopheles gambiae sensu lato. Bull. World Health Organ. 1999, 77, 230-234. [PubMed]

10. Diabaté, A.; Baldet, T.; Chandre, F.; Guiguemde, R.; Brengues, C.; Guillet, P.; Hemingway, J.; Hougard, J. First report of the kdr mutation in Anopheles gambiae M form from Burkina Faso, West Africa. Parassitologia 2002, 44, 157-158. [PubMed]

11. Awolola, T.S.; Oyewole, I.O.; Amajoh, C.N.; Idowu, E.T.; Ajayi, M.B.; Oduola, A.; Manafa, O.U.; Ibrahim, K.; Koekemoer, L.L.; Coetzee, M. Distribution of the molecular forms of Anopheles gambiae and pyrethroid knock down resistance gene in Nigeria. Acta Trop. 2005, 95, 204-209. [CrossRef] [PubMed]

12. Fanello, C.; Santolamazza, F.; Della-Torre, A. Simultaneous identification of species and molecular forms of the Anopheles gambiae complex by PCR-RFLP. Med. Vet. Entomol. 2002, 16, 461-464. [CrossRef] [PubMed]

13. Weill, M.; Chandre, F.; Brengues, C.; Manguin, S.; Akogbeto, M.; Pasteur, N. The kdr mutation occurs in the Mopti form of Anopheles gambiae s.s. through introgression. Insect Mol. Bol. 2000, 9, 451-455. [CrossRef]

14. Della-Torre, A.; Fanello, C.; Akogbeto, M.; Favia, G.; Petrarca, V.; Coluzzi, M. Molecular evidence of incipient speciation within Anopheles gambiae s.s. in West Africa. Insect Mol. Bol. 2001, 10, 9-18. [CrossRef]

15. N'Guessan, R.N.; Corbel, V.; Akogbéto, M.; Rowland, M. Reduced Efficacy of Insecticide- treated Nets and Indoor Residual Spraying for Malaria Control in Pyrethroid Resistance Area, Benin. Emerg. Infect. Dis. 2007, 13, 199-206. [CrossRef] [PubMed]

16. Diabaté, A.; Baldet, T.; Chandre, F.; Akogbeto, M.; Guiguemde, T.; Darriet, F.; Brengues, C.; Small, G.; Hougard, J. The role of agricultural use of insecticides in resistance to pyrethroids in Anopheles gambiae s.l. in Burkina Faso. Am. J. Trop. Med. Hyg. 2002, 67, 617-622. [CrossRef] [PubMed]

17. Verhaeghen, K.; Van Bortel, W.; Roelants, P.; Backeljau, T.; Coosemans, M. Detection of the East and West African kdr mutation in Anopheles gambiae and Anopheles arabiensis from Uganda using a new assay based on FRET/Melt Curve analysis. Malar. J. 2006, 9, 1-9. [CrossRef]

18. Etang, J.; Fondjo, E.; Chandre, F.; Morlais, I.; Brengues, C.; Nwane, P.; Chouaibou, M.; Ndjemai, H.; Frédéric, S. Short report: First report of knockdown mutations in the malaria vector Anopheles gambiae from cameroon. Am. J. Trop. Med. Hyg. 2006, 74, 795-797. [PubMed]

19. Nwane, P.; Etang, J.; Chouaibou, M.; Toto, J.; Koffi, A.; Mimpfound, R.; Simard, F. Multiple insecticide resistance mechanisms in Anopheles gambiae s.l. populations from Cameroon, Central Africa. Parasites Vectors 2013, 6, 41. [CrossRef] [PubMed] 
20. Djégbe, I.; Boussari, O.; Sidick, A.; Martin, T.; Ranson, H.; Chandre, F.; Akogbéto, M.; Corbel, V. Dynamics of insecticide resistance in malaria vectors in Benin: First evidence of the presence of L1014S kdr mutation in Anopheles gambiae from West Africa. Malar. J. 2011, 10, 261. [CrossRef] [PubMed]

21. Padonou, G.; Sezonlin, M.; Ossè, R.; Aïzoun, N.; Agbo, F.; Oussou, O.; Gbédjissi, G.; Akogbéto, M. Impact of three years of large scale indoor residual spraying (IRS) and insecticide treated nets (ITNs) interventions on insecticide resistance in Anopheles gambiae s.l. in Benin. Parasites Vectors 2012, 5, 72. [CrossRef] [PubMed]

22. Zoubiri, S.; Baaliouamer, A. Potentiality of plants as source of insecticide principles. J. Saudi Chem. Soc. 2014, 18, 925-938. [CrossRef]

23. Tripathi, A.K.; Upadhyay, S.; Bhuiyan, M.; Bhattacharya, P.R. A review on prospects of essential oils as biopesticide in insect-pest management. J. Pharmacogn. Phyther. 2009, 1, 52-63.

24. Isman, M.B.; Miresmailli, S.; Machial, C. Commercial opportunities for pesticides based on plant essential oils in agriculture, industry and consumer products. Phytochem. Rev. 2011, 10, 197-204. [CrossRef]

25. Rehman, J.U.; Ali, A.; Khan, I.A. Fitoterapia Plant based products: Use and development as repellents against mosquitoes: A review. Fitoterapia 2014, 95, 65-74. [CrossRef] [PubMed]

26. Gnankiné, O. Use of biopesticides in the perspective of chemical resistance management in Western Africa: The cases of Bemisia tabaci (Homotera: Aleyrodidae) and Anopheles gambiae (Diptera: Culicidae). Trends Entomol. 2012, 8, 85-95.

27. Nerio, S.L.; Olivero-verbel, J.; Stashenko, E. Bioresource Technology Repellent activity of essential oils: A review. Bioresour. Technol. 2010, 101, 372-378. [CrossRef] [PubMed]

28. Katz, T.; Miller, J.H.; Hebert, A. Insects repellents: Historical perspectives and new developments. J. Am. Acad. Dermatol. 2008, 58, 865-871. [CrossRef] [PubMed]

29. Guenther, E. The Essential Oils; D. Van Nostrand Co. Inc.: New York, NY, USA, 1948; p. 214.

30. ASTA. Official Analytical Methods of the American Spice Trade Association; ASTA: Englewood Cliffs, NJ, USA, 1968.

31. Chialva, F.; Gabri, G.; Liddle, P.A.P.; Ulian, F. Qualitative evaluation of aromatic herbs by direct headspace GC analysis. Application of the method and comparison with the traditional analysis of essential oil. J. High Resolut. Chromatogr. 1982, 5, 182-188. [CrossRef]

32. Burbott, A.J.; Loomis, W.D. Effects of light and temperature on the monoterpenes of peppermint. Plant Physiol. 1967, 42, 20-28. [CrossRef] [PubMed]

33. Takeoka, G.; Ebeler, S.; Jennings, W. Capillary gas chromatographic analysis of volatile flavor compounds. In American Chemical Society Symposium; American Chemical Society: Washington, DC, USA, 1985; pp. 96-108.

34. Bakkali, F.; Averbeck, S.; Averbeck, D.; Idaomar, M. Biological effects of essential oils-A review. Food Chem. Toxicol. 2008, 46, 446-475. [CrossRef] [PubMed]

35. Glasby, J. Encyclopédia of the Terpenoids; Wiley: New York, NY, USA, 1982; pp. 125-187.

36. Prajapati, V.; Tripathi, A.K.; Aggarwal, K.K.; Khanuja, S.P.S. Insecticidal, repellent and oviposition-deterrent activity of selected essential oils against Anopheles stephensi, Aedes aegypti and Culex quinquefasciatus. Bioresour. Technol. 2005, 96, 1749-1757. [CrossRef] [PubMed]

37. Bassolé, I.; Guelbeogo, W.; Nébié, R.; Costantini, C.; Sagnon, N.; Kaboré, Z.; Traoré, S. Ovicidal and larvicidal activity against Aedes aegypti and Anopheles gambiae complex mosquitoes of essential oils extracted from three spontaneous plants of Burkina Faso. Parassitologia 2003, 45, 23-26. [PubMed]

38. Tchoumbougnang, F.; Dongmo, P.; Sameza, L.; Mbanjo, N.; Fotso, G.; Zollo, P.; Menut, C. Activité larvicide sur Anopheles gambiae Giles et composition chimique des huiles essentielles extraites de quatre plantes cultivées au Cameroun. Biotechnol. Agron. Soc. Environ. 2009, 13, 77-84.

39. Deletre, E.; Martin, T.; Campagne, P.; Bourguet, D.; Cadin, A.; Menut, C.; Bonafos, R.; Chandre, F. Repellent, Irritant and Toxic Effects of 20 Plant Extracts on Adults of the Malaria Vector Anopheles gambiae Mosquito. PLoS ONE 2013, 8. [CrossRef] [PubMed]

40. Govindarajan, M.; Rajeswary, M.; Senthilmurugan, S.; Vijayan, P.; Alharbi, N.S.; Km, S.; Khaled, J.M.; Benelli, G. Larvicidal activity of the essential oil from Amomum subulatum Roxb.(Zingiberaceae) against Anopheles subpictus, Aedes albopictus and Culex tritaeniorhynchus (Diptera: Culicidae), and non-target impact on four mosquito natural enemies. Physiol. Mol. Plant Pathol. 2017. [CrossRef]

41. Pitasawat, B.; Champakaew, D.; Choochote, W.; Jitpakdi, A.; Chaithong, U. Aromatic plant-derived essential oil: An alternative larvicide for mosquito control. Fitoterapia 2007, 78, 205-210. [CrossRef] [PubMed] 
42. Zhu, L.; Tian, Y. Chemical composition and larvicidal activity of essential oil of Artemisia gilvescens against Anopheles anthropophagus. Parsitol. Res. 2013, 1137-1142. [CrossRef] [PubMed]

43. Sanei-dehkordi, A.; Vatandoost, H.; Abaei, M.R. Chemical Composition and Larvicidal Activity of Bunium persicum Essential Oil Against Two Important Mosquitoes Vectors. J. Essen. Oil Bear. Plants 2016, 19, $349-357$. [CrossRef]

44. Massebo, F.; Tadesse, M.; Bekele, T.; Balkew, M.; Gebre-michael, T. Evaluation on larvicidal effects of essential oils of some local plants against Anopheles arabiensis Patton and Aedes aegypti Linnaeus (Diptera, Culicidae) in Ethiopia. Afr. J. Biotechnol. 2009, 8, 4183-4188.

45. Kiran, S.R.; Bhavani, K.; Devi, P.S.; Rao, B.R.R.; Reddy, K.J. Composition and larvicidal activity of leaves and stem essential oils of Chloroxylon swietenia DC against Aedes aegypti and Anopheles stephensi. Bioresour. Technol. 2006, 97, 2481-2484. [CrossRef] [PubMed]

46. Mozaffari, E.; Abai, M.R.; Khanavi, M.; Vatandoost, H.; Sedaghat, M.M.; Moridnia, A.; Saber-Navaei, M.; Sanei-Dehkordi, A.; Rafi, F. Chemical composition, larvicidal and repellency properties of Cionura erecta (L.) Griseb. against malaria vector, Anopheles stephensi liston (Diptera: Culicidae). J. Arthropod-Borne Dis. 2014, 8, 147-155. [PubMed]

47. Sanei-dehkordi, A.; Sedaghat, M.M.; Vatandoost, H. Original Article Chemical Compositions of the Peel Essential Oil of Citrus aurantium and Its Natural Larvicidal Activity against the Malaria Vector Anopheles stephensi (Diptera: Culicidae) in Comparison with Citrus paradisi. J. Arthropod-Borne Dis. 2016, 10, 577-585. [PubMed]

48. Govindarajan, M.; Sivakumar, R. Mosquito larvicidal activity of thymol from essential oil of Coleus aromaticus Benth. against Culex tritaeniorhynchus, Aedes albopictus, and Anopheles subpictus (Diptera: Culicidae). Parasitol. Res. 2013, 112, 3713-3721. [CrossRef] [PubMed]

49. Mdoe, F.P.; Cheng, S.; Lyaruu, L.; Nkwengulila, G.; Chang, S.; Kweka, E.J. Larvicidal efficacy of Cryptomeria japonica leaf essential oils against Anopheles gambiae. Parasites Vectors 2014, 7, 1-6. [CrossRef] [PubMed]

50. Ali, A.; Wang, Y.; Khan, I.A. Larvicidal and Biting Deterrent Activity of Essential Oils of Curcuma longa, Ar-turmerone, and Curcuminoids Against Aedes aegypti and Anopheles quadrimaculatus (Culicidae: Diptera). J. Med. Entomol. 2015, 1-8. [CrossRef]

51. Ntonga, P.A.; Baldovini, N.; Mouray, E.; Mambu, L.; Belong, P.; Grellier, P. Activity of Ocimum basilicum, Ocimum canum, and Cymbopogon citratus essential oils against Plasmodium falciparum and mature-stage larvae of Anopheles funestus s. s. Parasite 2014, 21, 33. [CrossRef] [PubMed]

52. Senthilkumar, A.; Jayaraman, M.; Venkatesalu, V. Chemical constituents and larvicidal potential of Feronia limonia leaf essential oil against Anopheles stephensi, Aedes aegypti and Culex quinquefasciatus. Parsitol. Res. 2013, 1337-1342. [CrossRef] [PubMed]

53. Golfakhrabadi, F.; Khanavi, M.; Ostad, S.N.; Saeidnia, S. Original Article Biological Activities and Composition of Ferulago carduchorum Essential Oil. J. Arthropod Borne Dis. 2015, 9, 104-115. [PubMed]

54. Karunamoorthi, K.; Girmay, A.; Hayleeyesus, S.F. Mosquito repellent activity of essential oil of Ethiopian ethnomedicinal plant against Afro-tropical malarial vector Anopheles arabiensis. J. King Saud Univ. Sci. 2014, 26, 305-310. [CrossRef]

55. Kulkarni, R.R.; Pawar, P.V.; Joseph, M.P.; Akulwad, A.K.; Sen, A.; Joshi, S.P. Lavandula gibsoni and Plectranthus mollis essential oils: Chemical analysis and insect control activities against Aedes aegypti, Anopheles stephensi and Culex quinquefasciatus. J. Pest Sci. 2013, 86, 713-718. [CrossRef]

56. Govindarajan, M.; Sivakumar, R.; Rajeswary, M.; Yogalakshmi, K. Experimental Parasitology Chemical composition and larvicidal activity of essential oil from Ocimum basilicum (L.) against Culex tritaeniorhynchus, Aedes albopictus and Anopheles subpictus (Diptera: Culicidae). Exp. Parasitol. 2013, 134, 7-11. [CrossRef] [PubMed]

57. Tripathi, A.K.; Prajapati, V.; Ahmad, A.; Aggarwal, K.K.; Khanuja, S.P.S. Piperitenone Oxide as Toxic, Repellent, and Reproduction Retardant Toward Malarial Vector Anopheles stephensi (Diptera: Anophelinae) J. Med. Entomol. 2004, 41, 691-698. [CrossRef]

58. Krishnamoorthy, S.; Chandrasekaran, M. Identification of chemical constituents and larvicidal activity of essential oil from Murraya exotica L. (Rutaceae) against Aedes aegypti, Anopheles stephensi and Culex quinquefasciatus (Diptera: Culicidae). Parasitol. Res. 2015, 114, 1839-1845. [CrossRef] [PubMed] 
59. Govindarajan, M.; Rajeswary, M.; Arivoli, S. Larvicidal and repellent potential of Zingiber nimmonii (J. Graham) Dalzell (Zingiberaceae) essential oil: An eco-friendly tool against malaria, dengue, and lymphatic filariasis mosquito vectors? Parasitol. Res. 2016, 115, 1807-1816. [CrossRef] [PubMed]

60. Matasyoh, J.C.; Wathuta, E.M.; Kariuki, S.T.; Chepkorir, R. Journal of Asia-Paci fi c Entomology Chemical composition and larvicidal activity of Piper capense essential oil against the malaria vector, Anopheles gambiae. J. Asia Pac. Entomol. 2011, 14, 26-28. [CrossRef]

61. Kweka, J.E.; Senthilkumar, A.; Venkatesalu, V. Toxicity of essential oil from Indian borage on the larvae of the African malaria vector mosquito, Anopheles gambiae. Parasites Vectors 2012, 5, 277. [CrossRef] [PubMed]

62. Senthilkumar, A.; Venkatesalu, V. Chemical composition and larvicidal activity of the essential oil of Plectranthus amboinicus (Lour.) Spreng against Anopheles stephensi: A malarial vector mosquito. Parsitol. Res. 2010, 107, 1275-1278. [CrossRef] [PubMed]

63. Govindarajan, M.; Rajeswary, M.; Veerakumar, K.; Muthukumaran, U.; Hoti, S.L.; Mehlhorn, H.; Barnard, D.R. Novel synthesis of silver nanoparticles using Bauhinia variegata: A recent eco-friendly approach for mosquito control. Parasitol. Res. 2015, 115, 723-733. [CrossRef] [PubMed]

64. Ali, A.; Demirci, B.; Kiyan, H.T.; Bernier, U.R.; Tsikolia, M.; Wedge, D.E.; Khan, I.A.; Husnu, K.; Bas, C.A.N. Biting Deterrence, Repellency, and Larvicidal Activity of Ruta chalepensis (Sapindales: Rutaceae) Essential Oil and Its Major Individual Constituents Against Mosquitoes. J. Med. Entomol. 2013, 50, 1267-1274. [CrossRef] [PubMed]

65. Govindarajan, M.; Benelli, G. $\alpha$-Humulene and $\beta$-elemene from Syzygium zeylanicum (Myrtaceae) essential oil: Highly effective and eco-friendly larvicides against Anopheles subpictus, Aedes albopictus, and Culex tritaeniorhynchus (Diptera: Culicidae). Parasitol. Res. 2016, 115, 2771-2778. [CrossRef] [PubMed]

66. Dharmagadda, V.S.S.; Naik, S.N.; Mittal, P.K.; Vasudevan, P. Larvicidal activity of Tagetes patula essential oil against three mosquito species. Bioresour. Technol. 2005, 96, 1235-1240. [CrossRef] [PubMed]

67. Liu, X.C.; Dong, H.W.; Zhou, L.; Du, S.S.; Liu, Z.L. Essential oil composition and larvicidal activity of Toddalia asiatica roots against the mosquito Aedes albopictus (Diptera: Culicidae). Parasitol. Res. 2013, 112, 1197-1203. [CrossRef] [PubMed]

68. Pandey, S.K.; Upadhyay, S.; Tripathi, A.K. Insecticidal and repellent activities of thymol from the essential oil of Trachyspermum ammi (Linn) Sprague seeds against Anopheles stephensi. Parasitol. Res. 2009, 105, 507-512. [CrossRef] [PubMed]

69. Tiwary, M.; Naik, S.N.; Tewary, D.K.; Mittal, P.K.; Yadav, S. Chemical composition and larvicidal activities of the essential oil of Zanthoxylum armatum DC (Rutaceae) against three mosquito vectors. J. Vector Borne Dis. 2007, 44, 198-204. [PubMed]

70. Sanei-dehkordi, A.; Soleimani-ahmadi, M.; Akbarzadeh, K.; Abadi, Y.S.; Paksa, A.; Gorouhi, M.A. Chemical Composition and Mosquito Larvicidal Properties of Essential Oil from Leaves of an Iranian Indigenous Plant Zhumeria majdae. J. Essen. Oil Bear. Plant. 2016, 19, 1454-1461. [CrossRef]

71. Kweka, E.J.; Lima, T.C.; Marciale, C.M.; Sousa, D.P. De Asian Paci fi c Journal of Tropical Biomedicine. Asian Pac. J. Trop. Biomed. 2016, 6, 290-294. [CrossRef]

72. Govindarajan, M.; Mathivanan, T.; Elumalai, K. Mosquito larvicidal, ovicidal, and repellent properties of botanical extracts against Anopheles stephensi, Aedes aegypti, and Culex quinquefasciatus (Diptera: Culicidae). Parsitol. Res. 2011, 109, 353-367. [CrossRef] [PubMed]

73. Govindarajan, M.; Benelli, G. Facile biosynthesis of silver nanoparticles using Barleria cristata: Mosquitocidal potential and biotoxicity on three non-target aquatic organisms. Parsitol. Res. 2015, 115, 925-935. [CrossRef] [PubMed]

74. Pavela, R.; Pavela, R. Essential oils for the development of eco-friendly mosquito larvicides: A review. Ind. Crop. Prod. 2016, 76, 174-187. [CrossRef]

75. Dias, C.N.; Moraes, F.D.C. Essential oils and their compounds as Aedes aegypti L. (Diptera: Culicidae) larvicides: Review. Parsitol. Res. 2014, 113, 565-592. [CrossRef] [PubMed]

76. Norris, E.J.; Gross, A.D.; Dunphy, B.M.; Bessette, S. Comparison of the Insecticidal Characteristics of Commercially Available Plant Essential Oils Against Aedes aegypti and Anopheles gambiae (Diptera: Culicidae). J. Med. Entomol. 2015, 52, 993-1002. [CrossRef] [PubMed]

77. Bossou, A.D.; Mangelinckx, S.; Yedomonhan, H.; Boko, P.M.; Akogbeto, M.C.; De Kimpe, N.; Avlessi, F.; Sohounhloue, D.C.K. Chemical composition and insecticidal activity of plant essential oils from Benin against Anopheles gambiae (Giles). Parasites Vectors 2013, 6, 337. [CrossRef] [PubMed] 
78. WHO. Test Procedures; World Health Organization: Geneva, Switzerland, 2013.

79. Choochote, W.; Chaithong, U.; Kamsuk, K.; Jitpakdi, A.; Tippawangkosol, P.; Tuetun, B.; Champakaew, D.; Pitasawat, B. Repellent activity of selected essential oils against Aedes aegypti. Fitoterapia 2007, 78, 359-364. [CrossRef] [PubMed]

80. Deletre, E.; Schatz, B.; Chandre, F.; Ratnadass, A. Prospects for repellent in pest control: Current developments and future challenges. Chemoecology 2016, 26, 127-142. [CrossRef]

81. Amer, A.; Mehlhorn, H. Repellency effect of forty-one essential oils against Aedes, Anopheles, and Culex mosquitoes. Parasitol. Res. 2006, 99, 478-490. [CrossRef] [PubMed]

82. WHO. Report of the Who Informal Consultation on the Evaluation and Testing of Insecticides CTD/WHOPES/IC/96.1 Geneva: Control of Tropical Diseases; World Health Organization: Geneva, Switzerland, 1996.

83. Rajikumar, S.; Jebanessan, A. Repellent activity of selected plant essential oils against the malarial fever mosquito Anopheles stephensi. Trop. Biomed. 2007, 24, 71-75.

84. Phasomkusolsil, S.; Soonwera, M. Comparative mosquito repellency of essential oils against Aedes aegypti (Linn.), Anopheles dirus (Peyton and Harrison) and Culex quinquefasciatus (Say). Asian Pac. J. Trop. Biomed. 2011, 1, S113-S118. [CrossRef]

85. Abagli, A.Z.; Alavo, T.B.C. Essential Oil from Bush Mint, Hyptis suaveolens, is as Effective as DEET for Personal Protection against Mosquito Bites. Open Entomol. 2011, 5, 45-48. [CrossRef]

86. Ipek, E.; Zeytinoglu, H.; Okay, S.; Tuylu, B.A.; Kurkcuoglu, M.; Baser, K.H.C. Food Chemistry Genotoxicity and antigenotoxicity of Origanum oil and carvacrol evaluated by Ames Salmonella/microsomal test. Food Chem. 2005, 93, 551-556. [CrossRef]

87. Govindarajan, M.; Sivakumar, R.; Rajeswari, M. Chemical composition and larvicidal activity of essential oil from Mentha spicata (Linn.) against three mosquito species. Parasitol. Res. 2012, 110, 2023-2032. [CrossRef] [PubMed]

88. Govindarajan, M.; Rajeswary, M.; Hoti, S.L.; Benelli, G. Research in Veterinary Science Larvicidal potential of carvacrol and terpinen-4-ol from the essential oil of Origanum vulgare (Lamiaceae) against Anopheles stephensi, Anopheles subpictus, Culex quinquefasciatus and Culex tritaeniorhynchus (Diptera: Culicidae). Res. Vet. Sci. 2016, 104, 77-82. [CrossRef] [PubMed]

89. Govindarajan, M.; Rajeswary, M.; Hoti, S.L.; Bhattacharyya, A.; Benelli, G. Eugenol, $\alpha$-pinene and $\beta$-caryophyllene from Plectranthus barbatus essential oil as eco-friendly larvicides against malaria, dengue and Japanese encephalitis mosquito vectors. Parasitol. Res. 2016, 115, 807-815. [CrossRef] [PubMed]

90. Deletre, E.; Chandre, F.; Williams, L.; Duménil, C.; Menut, C.; Martin, T. Electrophysiological and behavioral characterization of bioactive compounds of the Thymus vulgaris, Cymbopogon winterianus, Cuminum cyminum and Cinnamomum zeylanicum essential oils against Anopheles gambiae and prospects for their use as bednet treatmen. Parasites Vectors 2015, 8, 316. [CrossRef] [PubMed]

91. Jaenson, T.G.T.; Pålsson, K. Borg-karlson Anna-Karin Evaluation of Extracts and Oils of Mosquito (Diptera: Culicidae) Repellent Plants from Sweden and Guinea-Bissau. J. Med. Entomol. 2006, 43, 113-119. [CrossRef] [PubMed]

92. Sukumar, K.; Perich, M.; Boobar, L. Botanical derivatives in mosquito control: A review. J. Am. Mosq. Control Assoc. 1991, 7, 210-237. [PubMed]

93. Jantan, I.; Zaki, Z. Development of environment-friendly insect repellents from the leaf oils of selected malaysian plants. Rev. Biodivers. Environ. Conserv. 1999, 6, 1-7.

94. Park, B.-S.P.; Choi, W.-S.; Kim, J.-H.; Kim, K.-H.; Lee, S.-E. Monoterpenes from thyme (Thymus vulgaris) as potential mosquito repellents potential mosquito repellents. Source J. Am. Mosq. Control Assoc. 2005, 21, 80-83. [CrossRef]

95. Omolo, M.O.; Okinyo, D.; Ndiege, I.O.; Hassanali, A. Repellency of essential oils of some Kenyan plants against Anopheles gambiae. Phytochemistry 2004, 65, 2797-2802. [CrossRef] [PubMed]

96. Trongtokit, Y.; Rongsriyam, Y.; Komalamisra, N. Comparative Repellency of 38 Essential Oils against Mosquito Bites. Phyther. Res. 2005, 309, 303-309. [CrossRef] [PubMed]

97. Gillij, Y.G.; Gleiser, R.M.; Zygadlo, J.A. Mosquito repellent activity of essential oils of aromatic plants growing in Argentina. Bioresour. Technol. 2008, 99, 2507-2515. [CrossRef] [PubMed]

98. Odalo, J.O.; Omolo, M.O.; Malebo, H.; Angira, J.; Njeru, P.M.; Ndiege, I.O.; Hassanali, A. Repellency of essential oils of some plants from the Kenyan coast against Anopheles gambiae. Acta Trop. 2005, 95, $210-218$. [CrossRef] [PubMed] 
99. Pridham, J. Terpenoids in Plants; Academic Press: New York, NY, USA, 1967; pp. 59-82.

100. Davidson, P.; Parish, M. Methods for testing the efficacy of food antimicrobials. Food Technol. 1989, 43, 148-155.

101. Abbassy, M.; Abdelgaleil, S.; Rabie, R. Insecticidal and synergistic effects of Majorana hortensis essential oil and some of its major constituents. Entomol. Exp. Appl. 2009, 131, 225-232. [CrossRef]

102. Berenbaum, M.; Neal, J. Synergism between myristicin and xanthotoxin, a naturally cooccurring plant toxicant. J. Chem. Ecol. 1985, 11, 1349-1358. [CrossRef] [PubMed]

103. Regnault-Roger, C.; Vincent, C.; Arnason, J.T. Essential Oils in Insect Control: Low-Risk Products in a High-Stakes World. Annu. Rev. Entomol. 2012, 57, 405-424. [CrossRef] [PubMed]

104. Prates, H.T.; Santos, J.P.; Waquil, J.M.; Fabris, J.D.; Oliveira, A.B.; Foster, J.E.; Lagoas, S.; Horizonte, B. Insecticidal Activity of Monoterpenes Against Rhyzopertha dominica (F.) and Tribolium castaneum (Herbst). J. Stored Prod. Res. 1998, 34, 5-8. [CrossRef]

105. Regnault-Roger, C. The potential of botanical essential oils for insect pest control. Integr. Pest Manag. Rev. 1997, 34, 25-34. [CrossRef]

106. Rattan, R. Mechanism of action of insecticidal secondary metabolites of plant origin. Crop Prot. 2010, 29, 913-920. [CrossRef]

107. Fournier, D.; Mutero, A. Modification of acetylcholinesterase as a mechanism of resistance to insecticide. Comp. Biochem. Physiol. C Pharmacol. Toxicol. Endocrinol. 1994, 108, 19-31. [CrossRef]

108. Aygun, D.; Doganay, Z.; Altintop, L.; Guven, H.; Onar, M.; Deniz, T.; Sunter, T. Serum acetylcholinesterase and prognosis of acute organophosphate poisoning. J. Toxicol. Clin. Toxicol. 2002, 40, 903-910. [CrossRef] [PubMed]

109. Houghton, P.; Ren, Y.; Howes, M. Acetylcholinesterase inhibitors from plants and fungi. Nat. Prod. Rep. 2006, 23, 181-199. [CrossRef] [PubMed]

110. Abdelgaleil, S.A.; Mohamed, M.I.; Badawy, M.E.; El-arami, S.A. Fumigant and contact toxicities of monoterpenes to Sitophilus oryzae (L.) and Tribolium castaneum (Herbst) and their inhibitory effects on acetylcholinesterase activity. J. Chem. Ecol. 2009, 35, 518-535. [CrossRef] [PubMed]

111. Hideyuki, T.; Mitsuo, M. Inhibition of acetylcholinesterase activity by essential oil from Bergamot. Koryo Terupen oyobi Seiyu Kagaku ni Kansuru Toronkai Koen Yoshishu 2001, 45, 435-436.

112. Seo, S.M.; Jung, C.S.; Kang, J.; Lee, H.R.; Kim, S.W.; Hyun, J.; Park, I.K. Larvicidal and Acetylcholinesterase Inhibitory Activities of Apiaceae Plant Essential Oils and Their Constituents against Aedes albopictus and Formulation Development. J. Agric. Food Chem. 2015, 63, 9977-9986. [CrossRef] [PubMed]

113. Savelev, S.; Okello, E.; Perry, N.; Wilkins, R.; Perry, E. Synergistic and antagonistic interactions of anticholinesterase terpenoids in Salvia lavandulae folia essential oil. Pharmacol. Biochem. Behav. 2003, 75, 661-668. [CrossRef]

114. Keane, S.; Ryan, M. Purification, characterisation, and inhibition bymonoterpenes of acetylcholinesterase from the waxmoth, Galleria mellonella (L.). Insect Biochem. Mol. Biol. 1999, 29, 1097-1104. [CrossRef]

115. Evans, P. Multiple receptor types for octopamine in the locust. J. Physiol. 1981, 318, 99-122. [CrossRef] [PubMed]

116. Evans, P. Studies on the mode of action of octopamine, 5-hydroxytryptamine and proctolin on a myogenic rhythm in the locust. J. Exp. Biol. 1984, 110, 231-251. [PubMed]

117. Enan, E. Insecticidal activity of essential oils: Octopaminergic sites of action. Comp. Biochem. Physiol. 2001, 130, 325-337. [CrossRef]

118. Kostyukovsky, M.; Rafaeli, A.; Gileadi, C.; Demchenko, N.; Shaaya, E. Activation of octopaminergic receptors by essential oil constituents isolated from aromatic plants: Possible mode of action against insect pests. Pest Manag. Sci. 2002, 1106, 1101-1106. [CrossRef] [PubMed]

119. Evans, P.; Robb, S. Octopamine receptor subtypes and their modes of action. Neurochem. Res. 1993, 18, 869-874. [CrossRef] [PubMed]

120. Howell, K.; Evans, P. The characterization of presynaptic octopamine receptors modulating octopamine release from an identified neurone in the locust. J. Exp. Biol. 1998, 201, 2053-2060. [PubMed]

121. Bloomquist, J.R. Chloride channels as tools for developing selective insecticides. Arch. Insect Biochem. Physiol. 2003, 54, 145-156. [CrossRef] [PubMed] 
122. Tong, F.; Coats, J.R. Effects of monoterpenoid insecticides on $\left[{ }^{3} \mathrm{H}\right]-\mathrm{TBOB}$ binding in house fly GABA receptor and ${ }^{36} \mathrm{Cl}^{-}$À uptake in American cockroach ventral nerve cord. Pestic. Biochem. Physiol. 2010, 98, 317-324. [CrossRef]

123. Hold, M.; Sirisoma, S.; Ikeda, T.; Narahashi, T.; Casida, E. Thujone (the active component of absinthe): Aminobutyric acid type. A receptor modulation and metabolic detoxification. Proc. Natl. Acad. Sci. USA 2000, 97, 3826-3831. [CrossRef] [PubMed]

124. Ratra, G.; Casida, J. GABA receptor subunit composition relative to insecticide potency and selectivity. Toxicol. Lett. 2001, 122, 215-222. [CrossRef]

125. Priestley, C.; Williamson, E.; Wafford, K.; Satelle, D.B. Thymol, a constituent of thyme essential oil, is a positive allosteric modulator of human GABA receptors and a homo-oligomeric GABA receptor from Drosophila melanogaster. Br. J. Pharmacol. 2003, 140, 1363. [CrossRef] [PubMed]

126. Krimer, V.; Vaštag, Ž.; Radulovi, L.; Peri, I. Microencapsulation Technology and Essential Oil Pesticides for Food Plant Production. In Essential Oils in Food Preservation, Flavor and Safety; Preedy, V., Ed.; Elsevier: Amsterdam, The Netherlands, 2016; pp. 123-129.

127. Devi, N.; Maji, T.K. Neem seed oil: Encapsulation and controlled release-Search for a greener alternative for pest control. In Pesticides in the Modern World_Pesticides Use and Management; Stoytcheva, M., Ed.; InTech: Rijeka, Croatia, 2011; pp. 191-232.

128. Maji, T.; Baruah, I.; Dube, S.; Hussain, M. Microencapsulation of Zanthoxylum limonella oil (ZLO) in glutaraldehyde crosslinked gelatin for mosquito repellent application. Bioresour. Technol. 2007, 98, 840-844. [CrossRef] [PubMed]

129. Tawatsin, A.; Wratten, S.D.; Scott, R.R.; Thavara, U.; Techadamrongsin, Y. Repellency of volatile oils from plants against three mosquito vectors. J. Vector Ecol. 2001, 26, 76-82.

130. Specos, M.M.; Garcia, J.J.; Tornesello, J.; Marino, P.; Vecchia, M.D.; Tesoriero, M.D.; Hermida, L.G. Transactions of the Royal Society of Tropical Medicine and Hygiene Microencapsulated citronella oil for mosquito repellent finishing of cotton textiles. Trans. R. Soc. Trop. Med. Hyg. 2010, 104, 653-658. [CrossRef] [PubMed]

131. Solomon, B.; Sahle, F.; Gebre-Mariam, T.; Asres, K.; Neubert, R. Microencapsulation of citronella oil for mosquito-repellent application: Formulation and in vitro permeation studies. Eur. J. Pharm. Biopharm. 2012, 80, 61-66. [CrossRef] [PubMed]

132. Sakulkua, U.; Nuchuchuaa, O.; Uawongyartb, N.; Puttipipatkhachornc, S.; Soottitantawatd, A.; Ruktanonchaia, U. Characterization and mosquito repellent activity of citronella oil nanoemulsion. Int. J. Pharm. Nanotechnol. 2009, 372, 105-111. [CrossRef] [PubMed]

133. Duvallet, G.; De Gentile, L. Protection Personnelle Antivectorielle; IRD: Marseille, France, 2012; p. 352.

134. Zaim, M.; Aitio, A.; Nakashima, N. Safety of pyrethroid-treated mosquito nets. Med. Vet. Entomol. 2000, 14, 1-5. [CrossRef] [PubMed]

135. Palchick, S. The Biology of Disease of Disease Vectors; University Press of Colarado: Boulder, CO, USA, 1996.

136. McAllister, C.; Adams, M.F. Mode of Action for Natural Products Isolated From Essential Oils of Two Trees Is Different From Available Mosquito Adulticides. J. Med. Entomol. 2010, 47, 1123-1126. [CrossRef] [PubMed]

137. Chitwood, D. Phytochemical based strategies for nematode control. Annu. Rev. Phytopathol. 2002, 40, 221-249. [CrossRef] [PubMed]

138. Yadouleton, A.; Martin, T.; Padonou, G.; Chandre, F.; Asidi, A.; Djogbenou, L.; Dabiré, R.; Aïkpon, R.; Boko, M.; Glitho, I.; et al. Cotton pest management practices and the selection of pyrethroid resistance in Anopheles gambiae population in Northern Benin. Parasites Vectors 2011, 4, 60. [CrossRef] [PubMed]

(C) 2017 by the authors. Licensee MDPI, Basel, Switzerland. This article is an open access article distributed under the terms and conditions of the Creative Commons Attribution (CC BY) license (http:// creativecommons.org/licenses/by/4.0/). 